\title{
Synthesis and antifungal activity of some 2-benzothiazolylthioacetyl amino acid and peptide derivatives ${ }^{1}$
}

\author{
A. Aboelmagd, ${ }^{a}$ Ibrahim A. I. Ali, ${ }^{a}$ Ezzeldin M. S. Salem, ${ }^{\text {a,* }}$ and M. Abdel-Razik ${ }^{b}$ \\ ${ }^{a}$ Department of Chemistry, Faculty of Science, Suez Canal University, Ismailia, 41511, Egypt \\ ${ }^{b}$ Department of Microbiology, Faculty of Science, Suez Canal University, Ismailia, 41511, Egypt \\ E-mail:ezz_salem@yahoo.com
}

\begin{abstract}
A series of benzothiazolylthioacetyl amino acid and peptide derivatives including glycoside, hydrazide and hydrazone moieties was synthesized with the aim of evaluating their antifungal activity. Their chemical structures were confirmed by ${ }^{1} \mathrm{H}-\mathrm{NMR}$, IR, mass spectrometry and elemental analyses. Out of the thirty two tested compounds, three derivatives have high activity as fluconazole at $100 \mathrm{ppm}$ and six at $1000 \mathrm{ppm}$ toward Candida albicans, whereas they were inactive toward Aspergillus flavus.
\end{abstract}

Keywords: 2-Mercaptobenzothiazole, peptides, glycosides, hydrazides, hydrazones, antifungal activity

\section{Introduction}

2-Substituted benzothiazoles and their derivatives have attracted much attention of chemists and pharmacologists because of their broad spectrum of biological activities and applications as accelerators in rubber vulcanization, antioxidants, dyes, polymers and photographic materials. ${ }^{1-4}$ 2-Mercaptobenzothiazole, the first known benzothiazole derivative, was found to be an effective antifungal agent. ${ }^{5-10}$ Some peptide antibiotics are known to exhibit remarkable antifungal activity, such as iturins, bacillomycins, mycosubtilin, pradimicins and many others. ${ }^{11-14}$ These observations prompted us to undertake systematic study of the synthesis and antifungal evaluation of some mercaptobenzothiazolyl amino acid and peptide derivatives. The 2mercaptobenzothiazole derivatives were synthesized as shown in Schemes 1 and 2. The selection of the amino acids L-serine, L-tyrosine and DL- threonine is based on their presence as major constituents of the antifungal antibiotics iturins $\mathrm{A}$ and $\mathrm{D}$, bacillomycins $\mathrm{L}, \mathrm{D}$ and $\mathrm{F}$ and

\footnotetext{
${ }^{1}$ This article has been presented in abbreviated form in The 14th International Electronic Conference on Synthetic Organic Chemistry (ECSOC-14), 1 - 30 November 2010.
} 
mycosubtilin. ${ }^{11}$ In addition, L-hydroxyproline is found in the antifungal antibiotic echinocandin $\mathrm{B}^{15}$ whereas L-methionine is one of the constituent amino acids of the antifungal compound AK3 , isolated from Synechocystis sp. ${ }^{16}$

\section{Results and Discussion}

\section{Chemistry}

In the present study, the 2-benzothiazolylthioacetyl amino acid ester derivatives $\mathbf{2 a - d}$ and $\mathbf{3}$ were prepared from the hydrazide $1{ }^{17}$ via the racemization-free azide coupling method. ${ }^{18,}{ }^{19}$ Treatment of hydrazide 1 with nitrous acid $\left(\mathrm{NaNO}_{2} / \mathrm{HCl}\right)$ at $-5{ }^{\circ} \mathrm{C}$ afforded the corresponding azide, which was isolated in ethyl acetate and washed at low temperature $\left(0^{\circ} \mathrm{C}\right)$, to suppress its transformation to the isocyanate derivative by Curtius rearrangement. It is known that isocyanates are reactive towards the amino components in the coupling step leading to the formation of the undesired byproduct urea derivative. ${ }^{20}$

The azide solution in ethyl acetate reacted with amino acid methyl esters hydrochloride, previously treated with triethylamine in ethyl acetate at low temperature, to produce 2benzothiazolylthioacetyl amino acid ester derivatives 2a-d and $\mathbf{3}$ in good yields, and their chemical structures were confirmed by ${ }^{1} \mathrm{H}$ - NMR and elemental analysis. The ${ }^{1} \mathrm{H}-\mathrm{NMR}$ spectra showed a doublet signal at $8.3 \mathrm{ppm}$ for the $\mathrm{NH}$ proton of the peptide bond (except for the hydroxyproline derivative 3), multiplet signals between 6.8 and $8.0 \mathrm{ppm}$ for the four aromatic protons, a multiplet signal at 4.5-5.0 ppm for the $\alpha-\mathrm{CH}$ proton of the amino acids, and singlet signal at $3.6 \mathrm{ppm}$ for the three protons of $-\mathrm{OCH}_{3}$ of the ester groups. Also, the geminal coupling between the two protons of the thioacetyl group $\mathrm{SCH}_{2} \mathrm{CO}$ appears at $3.8-4.3 \mathrm{ppm}$ as two doublet signals. The other signals for the amino acid side chains are reported in the Experimental part.

The dipeptide derivatives $\mathbf{6 a - g}$ and $\mathbf{7 a}, \mathbf{b}$ were prepared from their corresponding amino acid ester derivatives, 2a-d and 3, after conversion to hydrazides $\mathbf{4 a - d}$ and $\mathbf{5}$ by boiling with excess hydrazine hydrate in methanol. The ${ }^{1} \mathrm{H}$ - NMR, IR spectra, and elemental analyses of 4a-d and $\mathbf{5}$ confirmed their structures as shown from the data reported in the Experimental part. The dipeptide derivatives 6a-g and 7a, $\mathbf{b}$ were obtained by the azide-coupling method in 30-43\% yields (Scheme 1). The ${ }^{1} \mathrm{H}$ - NMR spectra revealed two doublets in the range $7.5-8.5 \mathrm{ppm}$ for the two NH protons of the peptide bonds, and two multiplets in the range $4.0-5.0 \mathrm{ppm}$ for the $\alpha$ $\mathrm{CH}$ protons of the two amino acids, in addition to several other signals corresponding to protons of the individual side chains, (see the Experimental part).

Some other derivatives bearing physiologically important functional groups, such as the acetylated derivative 8 , glycosides 10a, $\mathbf{b}$, the hydrazide derivative 12, and hydrazones 13a, $\mathbf{b}$, 14a-f, 15, have been prepared for comparison (Scheme 2). 


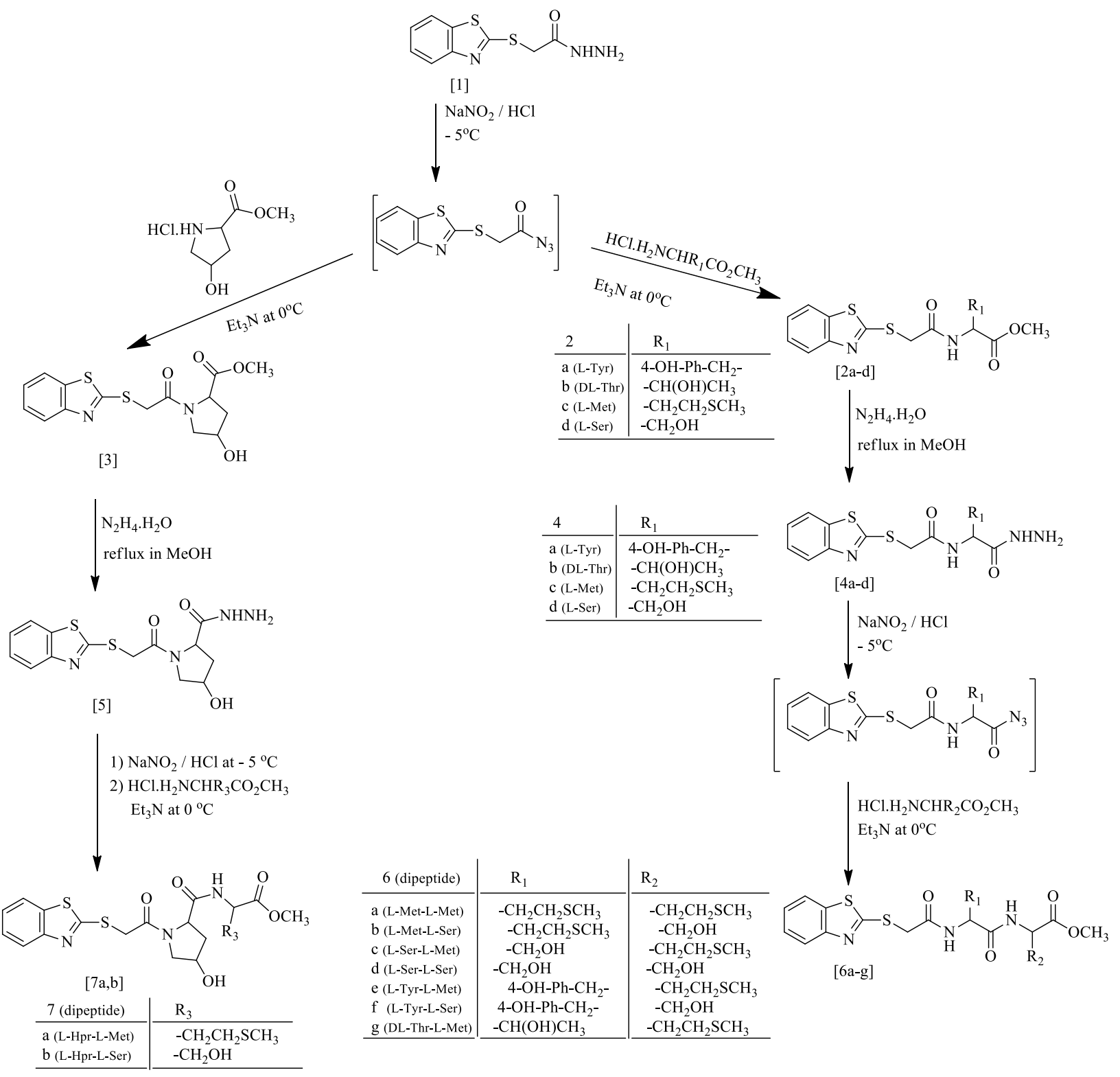

Scheme 1. Synthesis of benzothiazolylthioacetyl amino acid and peptide derivatives.

Acetylation of the hydroxyl group of the amino acid derivatives $\mathbf{2 a}$, $\mathbf{b}$, and $\mathbf{d}$ with acetic anhydride in the presence of pyridine failed to give pure products. However, exceptionally, compound $\mathbf{3}$ gave in good yield the acetyl derivative $\mathbf{8}$, which was easily purified by crystallization from ethyl acetate / petroleum ether. The ${ }^{1} \mathrm{H}$ NMR spectrum of $\mathbf{8}$ showed a singlet signal at $2.02 \mathrm{ppm}$ for the three protons of the acetyl group $\mathrm{COCH}_{3}$.

Next, our target was the synthesis of the glycopeptides $\mathbf{1 0 a}, \mathbf{b}$ by the procedure reported by Schmidt et al. ${ }^{21}$ Glycosylation of $\mathbf{2 d}$ and $\mathbf{3}$ as an alcohol-acceptor precursor, with $O$-(2:3, 5:6-diO-isopropylidene- $\alpha$-D-mannofuranose)trichloroacetimidate, $\mathbf{9}$ as donor precursor in the presence 
of catalytic amount of trimethylsilyl trifluoromethanesulfonate (TMSOTF) as Lewis acid afforded 10a,b in $35.8 \%$ and $28 \%$ yield, respectively.

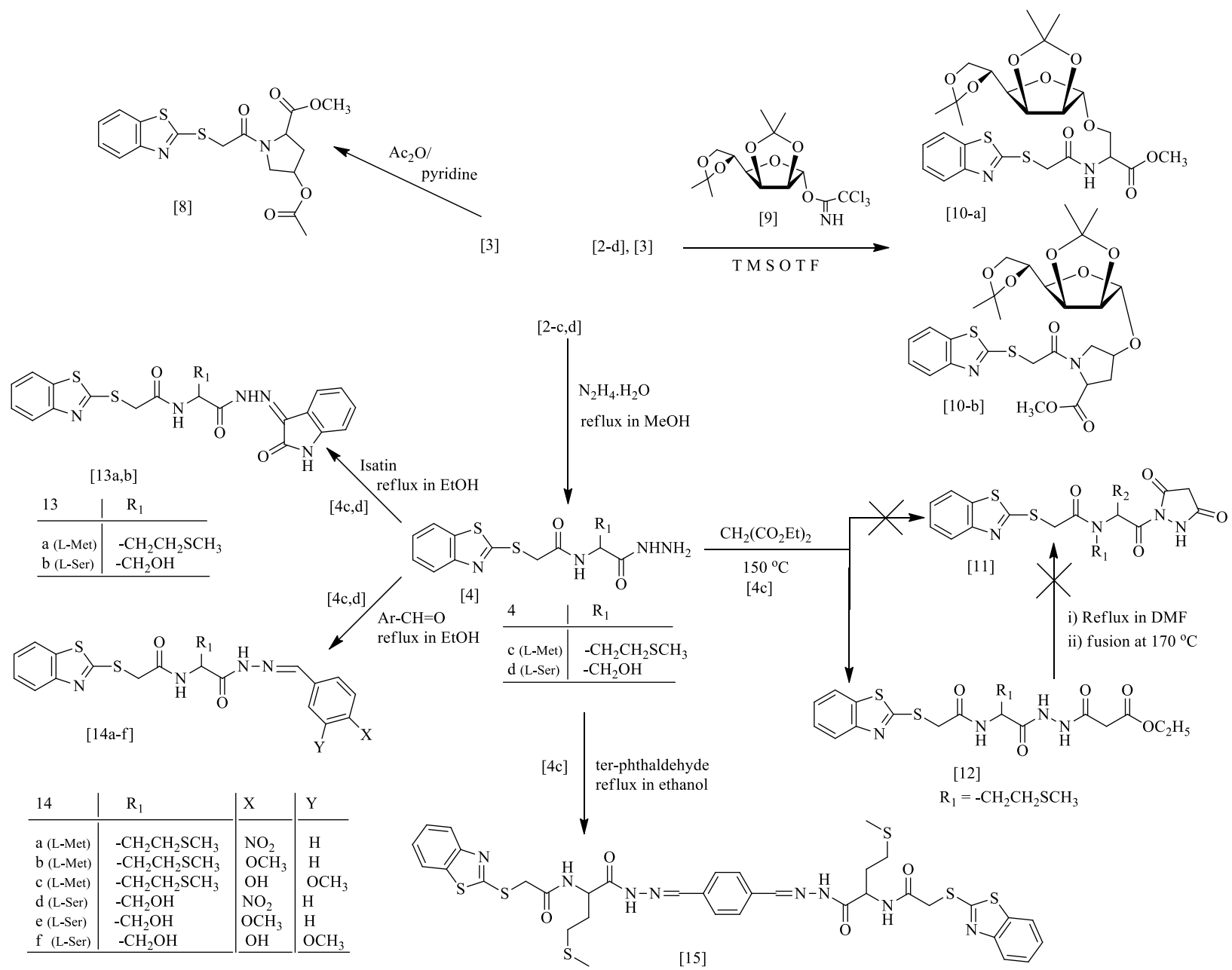

Scheme 2. Reactions of some 2-benzothiazolylthioacetyl amino acid derivatives.

The $O$-glycofuranosyl trichloroacetimidate 9 is characterized by its stability at room temperature for long periods, and gives the $\alpha$-anomer derivatives (Scheme 2). The structures of 10a,b were identified from the ${ }^{1} \mathrm{H}$ NMR and elemental analysis. In the ${ }^{1} \mathrm{H}$ NMR spectra the sugar protons appeared as a singlet at $5.34 \mathrm{ppm}$ for $\mathrm{H}-1$ of $\mathbf{1 0 a}$ and at $5.09 \mathrm{ppm}$ for $\mathrm{H}-1$ of $\mathbf{1 0 b}$, confirming the $\alpha$-anomer of the glycosides 10a,b. The doublet at $4.59 \mathrm{ppm}(J=5.8 \mathrm{~Hz})$ was attributed to $\mathrm{H}-2$, and a multiplet at 4.78-4.71 ppm was assigned for $\mathrm{H}-3$. The singlet at $3.68 \mathrm{ppm}$ and the four singlet signals at $1.41,1.33,1.29$ and $1.22 \mathrm{ppm}$ were assigned to the $-\mathrm{OCH}_{3}$ and 4$\mathrm{CH}_{3}$ of the isopropylidene groups, respectively.

Reaction of the hydrazide $\mathbf{4 c}$ with diethyl malonate led to the formation of the linear hydrazido-ethyl ester derivatives 12. The ${ }^{1} \mathrm{H}$ NMR spectrum of the product showed a singlet at $3.25 \mathrm{ppm}$ for (-CO-CH $2-\mathrm{CO}-)$, a multiplet signal at $4.12-4.06 \mathrm{ppm}$, and a triplet at $1.16 \mathrm{ppm}$ for 
the $-\mathrm{OCH}_{2} \mathrm{CH}_{3}$ group, indicative of the formation of the linear product $\mathbf{1 2}$ rather than the cyclic 11. Trials to obtain the corresponding pyrazolidinedione derivative $\mathbf{1 1}$ either by fusion of $\mathbf{1 2}$ or by heating in DMF were unsuccessful.

Hydrazone derivatives have gained importance due to their application in pharmaceutical chemistry. The biological activity associated with these compounds was attributed to the presence of the $(-\mathrm{CONHN}=\mathrm{CH}-)$ moiety. ${ }^{22}$ In the present study, some hydrazones have been synthesized by refluxing the hydrazides $\mathbf{4 c , d}$ with different carbonyl compounds such as isatin, $p$-nitrobenzaldehyde, anisaldehyde, vanillin and benzene-1,4-dicarboxaldehyde. The structures of the hydrazones 13a,b, 14a-f and 15 were investigated by IR, ${ }^{1} \mathrm{H}$ NMR, mass spectrometry and elemental analyses (see the Experimental part). The ${ }^{1} \mathrm{H}$ NMR spectra of hydrazones $\mathbf{1 4 a}, \mathbf{1 4 b}$ and 14c (Table 1) revealed the existence of a mixture of $E$ - and $Z$-stereoisomers (Fig. 1) in which the $E$-form predominates. This conclusion is supported by previously published data on similar compounds. $^{23,24}$

Table 1. Data of ${ }^{1} \mathrm{H}$ NMR spectra of compounds 14a-c

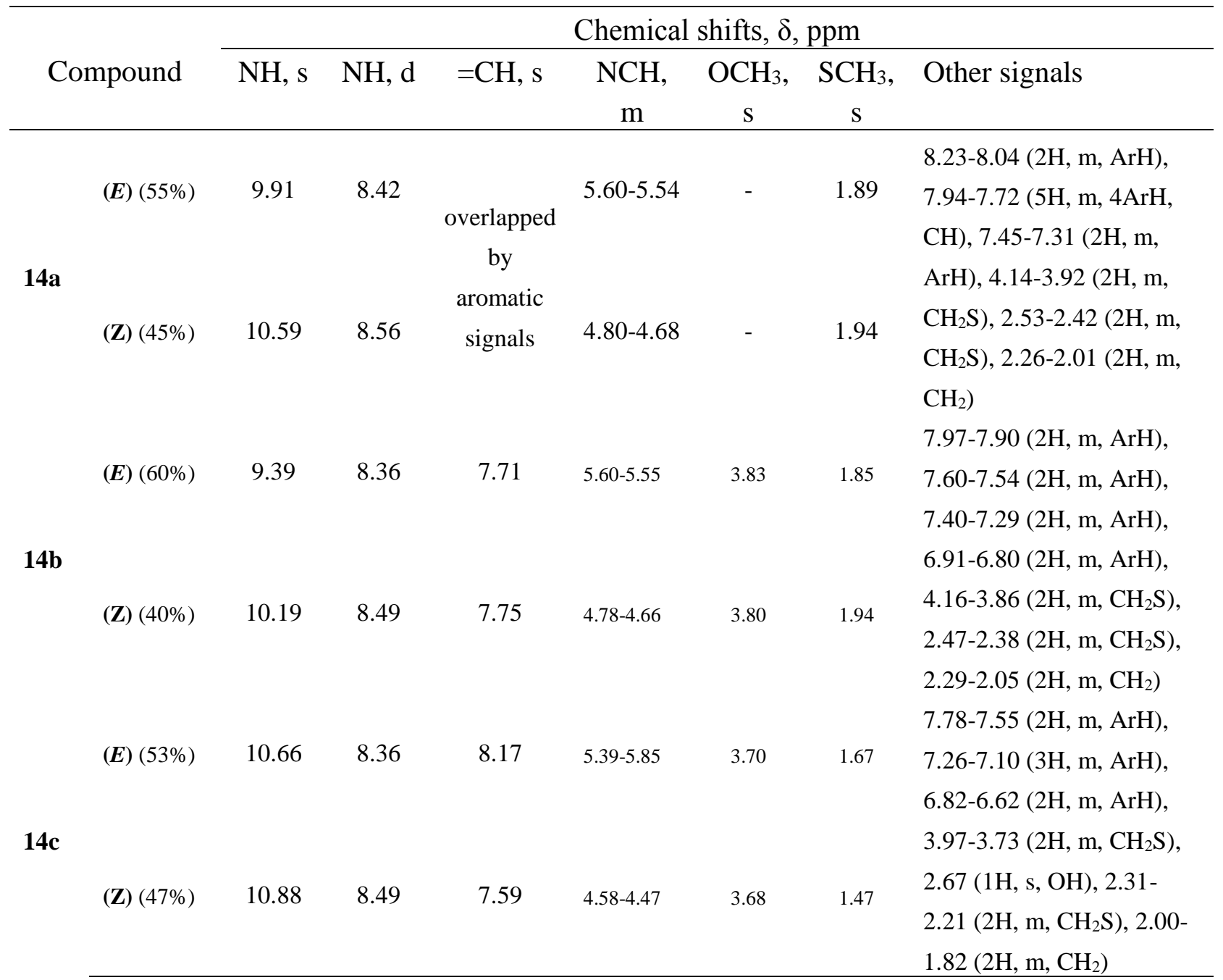



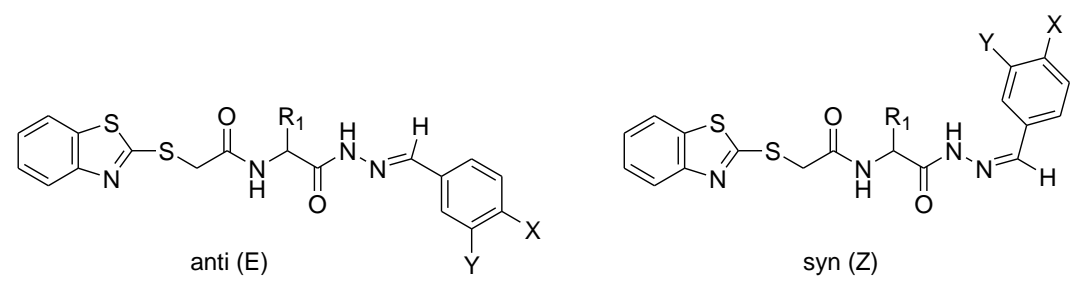

Figure 1. $E$ - and $Z$ - forms of compounds 14a-c.

\section{Biological evaluation}

The in vitro antifungal screening of the newly synthesized compounds against Aspergillus flavus and Candida albicans were carried out using fluconazole as standard, by the well technique of the agar diffusion method. The recorded data [Tables 2 and 3], showed that, out of the thirty two tested compounds three derivatives have activity comparable to fluconazole at $100 \mathrm{ppm}$, and six at $1000 \mathrm{ppm}$ toward Candida albicans. All the synthesized and tested benzothiazolyl derivatives were devoid of antifungal activity (except for 10a) against the mold Aspergillus flavus.

Table 2. The antifungal effects of the synthesized compounds against Aspergillus flavus and Candida albicans indicated by the diameter of the inhibition zones (well method)

\begin{tabular}{ccccccccc}
\hline \multirow{2}{*}{ Compd. } & \multicolumn{3}{c}{ Aspergillus flavus } & \multicolumn{5}{c}{ Candida albicans } \\
\cline { 2 - 7 } MBT & $100 \mathrm{ppm}$ & $300 \mathrm{ppm}$ & $500 \mathrm{ppm}$ & $1000 \mathrm{ppm}$ & $100 \mathrm{ppm}$ & $300 \mathrm{ppm}$ & $500 \mathrm{ppm}$ & $1000 \mathrm{ppm}$ \\
\hline $\mathbf{2 a}$ & -- & -- & + & ++ & + & + & ++ & +++ \\
$\mathbf{2 b}$ & -- & -- & -- & -- & + & + & ++ & +++ \\
$\mathbf{3}$ & -- & -- & -- & -- & -- & -- & -- & + \\
$\mathbf{4 c}$ & -- & -- & -- & -- & -- & + & + & +++ \\
$\mathbf{5}$ & -- & -- & -- & -- & -- & + & + & ++ \\
$\mathbf{6 f}$ & -- & -- & -- & -- & -- & -- & -- & ++ \\
$\mathbf{7 a}$ & -- & -- & -- & -- & -- & -- & -- & + \\
$\mathbf{7 b}$ & -- & -- & -- & + & -- & -- & -- & ++ \\
$\mathbf{8}$ & -- & -- & -- & + & -- & -- & -- & ++ \\
$\mathbf{1 0 a}$ & -- & -- & -- & -- & + & + & ++ & +++ \\
$\mathbf{1 0 b}$ & -- & + & + & ++ & -- & -- & + & ++ \\
$\mathbf{1 2}$ & -- & -- & -- & -- & -- & -- & -- & + \\
$\mathbf{1 3 a}$ & -- & -- & -- & -- & -- & -- & -- & + \\
$\mathbf{1 3 b}$ & -- & -- & -- & -- & -- & + & ++ & +++ \\
$\mathbf{1 4 a}$ & -- & -- & -- & -- & -- & + & ++ & +++ \\
$\mathbf{1 4}$ & -- & -- & -- & -- & + & + & + & ++ \\
$\mathbf{1 4 d}$ & -- & -- & -- & -- & -- & + & ++ & +++ \\
$\mathbf{1 5}$ & -- & -- & -- & -- & -- & -- & -- & + \\
Fluconazole & +- & -- & -- & -- & -- & -- & + & ++ \\
& + & ++ & +++ & +++ & + & ++ & +++ & +++ \\
\hline
\end{tabular}

Zone diameter of growth inhibition: (--) inactive, $(+)<10 \mathrm{~mm},(++) 10-15 \mathrm{~mm},(+++)>16 \mathrm{~mm}$. 
The tyrosine and $O$-acetyl hydroxyproline methyl ester derivatives $\mathbf{2 a}$ and $\mathbf{8}$, respectively, were the most potent compounds against Candida albicans among the tested series. Surprisingly, conversion of the benzothiazolyl amino acid ester derivatives to the corresponding hydrazides does not improve appreciably their antifungal activity. However, hydrazone formation led to higher activity than the corresponding hydrazides, especially those of isatin 13a, b and methionine 14a, c derivatives. In general all the tested dipeptide derivatives were actually inactive with exception of $\mathbf{7 a}, \mathbf{b}$, which showed slight activity against both Candida albicans and Aspergillus flavus.

Table 3. The MIC and the MFC of the synthesized compounds compared with MBT and fluconazole

\begin{tabular}{|c|c|c|c|c|}
\hline \multirow[b]{2}{*}{ Compd. } & \multicolumn{2}{|c|}{ Aspergillus flavus } & \multicolumn{2}{|c|}{ Candida albicans } \\
\hline & $\begin{array}{c}\text { MIC } \\
\text { values } \\
(\mu \mathrm{g} / \mathrm{ml})\end{array}$ & $\begin{array}{c}\text { MFC } \\
\text { values } \\
(\mu \mathrm{g} / \mathrm{ml})\end{array}$ & $\begin{array}{c}\text { MIC } \\
\text { values } \\
(\mu \mathrm{g} / \mathrm{ml})\end{array}$ & $\begin{array}{c}\text { MFC } \\
\text { values } \\
(\mu \mathrm{g} / \mathrm{ml})\end{array}$ \\
\hline MBT & 500 & -- & 100 & 500 \\
\hline $\mathbf{2 a}$ & -- & -- & 100 & 500 \\
\hline $2 \mathbf{b}$ & -- & -- & 1000 & -- \\
\hline 3 & -- & -- & 300 & -- \\
\hline $4 c$ & -- & -- & 300 & -- \\
\hline 5 & -- & -- & 1000 & -- \\
\hline 6f & -- & -- & 1000 & -- \\
\hline $7 \mathbf{a}$ & 1000 & -- & 1000 & -- \\
\hline $7 \mathbf{b}$ & 1000 & -- & 1000 & -- \\
\hline 8 & -- & -- & 100 & 500 \\
\hline $10 \mathbf{a}$ & 300 & 500 & 500 & 500 \\
\hline $10 b$ & -- & -- & 1000 & -- \\
\hline 12 & -- & -- & 1000 & -- \\
\hline 13a & -- & -- & 300 & 300 \\
\hline 13b & -- & -- & 300 & 500 \\
\hline $14 \mathbf{a}$ & -- & -- & 100 & 500 \\
\hline $14 c$ & -- & -- & 300 & -- \\
\hline $14 d$ & -- & -- & 1000 & -- \\
\hline 15 & -- & -- & 500 & -- \\
\hline Fluconazole & 100 & 300 & 100 & 300 \\
\hline
\end{tabular}

MIC: the minimum inhibitory concentration MFC: the minimum fungicidal concentration (--) inactive 


\section{Experimental Section}

General. The boiling point range of the petroleum used was $40-60{ }^{\circ} \mathrm{C}$. Thin layer chromatography (TLC) was carried out on silica gel $60 \mathrm{~F}_{254}$ plastic plates (E. Merck, layer thickness $0.2 \mathrm{~mm}$ ) in the following solvent systems:- $S_{1}$, chloroform: methanol (95: 5); $S_{2}$, chloroform: methanol (90: 10); $\mathrm{S}_{3}$, ethyl acetate: petroleum ether (2: 1). The spots on thin layer plates were detected by UV lamp. Melting points were determined on a Buchi 510 melting-point apparatus and the values are uncorrected. RT denotes room temperature.

Elemental analyses were performed on a Flash EA-1112 instrument at the Microanalytical Laboratory, Faculty of Science, Suez Canal University, Ismailia, Egypt. $\left({ }^{1} \mathrm{H}\right.$ NMR spectra were measured on Bruker (200 MHz) and TMS was used as internal standard, IR spectra were recorded on a Perking Elmer 1430 ratio recording infrared spectrophotometer with CDS data station using KBr Wafer technique, and Mass spectra were measured on a GC-MSQP 1000EX Shimadzu at microanalytical laboratory, Cairo University, Cairo, Egypt. The starting compound $\mathbf{1}$ was prepared according to the method described in the literature. ${ }^{17}$

\section{General procedure for preparation of compounds $\mathbf{2 a - d ,} 3$}

To a cold solution $\left(-5^{\circ} \mathrm{C}\right)$ of hydrazide $\mathbf{1}(0.8 \mathrm{mmol})$ in acetic acid $(6 \mathrm{ml})$, hydrochloric acid $(5 \mathrm{~N}$, $3 \mathrm{ml})$, and water $(25 \mathrm{ml})$, was added portionwise under stirring a cold solution $\left(0{ }^{\circ} \mathrm{C}\right)$ of sodium nitrite $(0.07 \mathrm{~g}, 1.0 \mathrm{mmol})$ in water $(3 \mathrm{ml})$. After stirring at the same temperature for 30 minutes, the azide was extracted with cold ethyl acetate, and washed successively with cold water, $5 \%$ $\mathrm{NaHCO}_{3}$, and water. After drying over anhydrous sodium sulphate, the azide was used without further purification in the next step. After stirring the reaction mixture of amino acid ester hydrochlorides $(0.9 \mathrm{mmol})$, triethylamine $(0.2 \mathrm{ml})$ in ethyl acetate $(30 \mathrm{ml})$ at $0{ }^{\circ} \mathrm{C}$ for 20 minutes, the formed triethylamine hydrochloride was filtered off. The previously prepared cold dried solution of the azide was added to the cold ethyl acetate solution of the amino acid ester. The mixture was kept $12 \mathrm{hrs}$ in the refrigerator and then at room temperature for an additional $12 \mathrm{hrs}$. The reaction mixture was washed with $0.1 \mathrm{~N} \mathrm{HCl}$, water, $5 \% \mathrm{NaHCO}_{3}$, water and then dried over anhydrous sodium sulphate. The solvent was evaporated in vacuum and the residue was crystallized from ethyl acetate-petroleum ether to give the 2-benzothiazol-ylthioacetyl amino acid esters $\mathbf{2 a - d}$ and $\mathbf{3}$.

2-Benzothiazolylthioacetyl L-tyrosine methyl ester (2a). Colorless crystals $(0.76 \mathrm{~g}, 47.5 \%)$, $\mathrm{R}_{\mathrm{f}}$ $=0.27\left(\mathrm{~S}_{1}\right), \mathrm{mp} 128-131{ }^{\circ} \mathrm{C} .{ }^{1} \mathrm{H}$ NMR $\left(\mathrm{CDCl}_{3}\right): \delta=8.24(1 \mathrm{H}, \mathrm{d}, J=8.0 \mathrm{~Hz}, \mathrm{NH}), 7.89-7.20(4 \mathrm{H}$, $\mathrm{m}, \operatorname{ArH}), 6.91(2 \mathrm{H}, \mathrm{m}, \mathrm{ArH}), 6.71(2 \mathrm{H}, \mathrm{m}, \mathrm{ArH}), 4.91(1 \mathrm{H}, \mathrm{m}, \mathrm{NCH}), 4.30(1 \mathrm{H}, \mathrm{s}, \mathrm{OH}), 4.10$ $\left(1 \mathrm{H}, \mathrm{d}, J_{\mathrm{gem}}=14.6 \mathrm{~Hz}, \mathrm{SCH}\right), 3.82\left(1 \mathrm{H}, \mathrm{d}, J_{\text {gem }}=14.6 \mathrm{~Hz}, \mathrm{SCH}\right), 3.63\left(3 \mathrm{H}, \mathrm{s}, \mathrm{OCH}_{3}\right), 3.08-2.98$ (2H, m, $\mathrm{CH}_{2} \mathrm{Ph}$ ). Anal. Calcd. for $\mathrm{C}_{19} \mathrm{H}_{18} \mathrm{~N}_{2} \mathrm{O}_{4} \mathrm{~S}_{2}$ (402.48): C, 56.69; H, 4.51; N, 6.96; Found C, 56.50; H, 4.59; N, 7.29. Mass spectrum, $m / z$ (\%): 402 (9.3), 225 (58.3), 208 (100), 180 (51.2), 167 (57.2), 135 (32.2), 107 (83.6). 
2-Benzothiazolylthioacetyl DL-threonine methyl ester (2b). Colorless crystals (0.86 g, 63.7\%), $\mathrm{R}_{\mathrm{f}}=0.60\left(\mathrm{~S}_{1}\right), \mathrm{mp} 88-91{ }^{\circ} \mathrm{C} .{ }^{1} \mathrm{H} \mathrm{NMR}\left(\mathrm{CDCl}_{3}\right): \delta=8.32(1 \mathrm{H}, \mathrm{d}, J=7.6 \mathrm{~Hz}, \mathrm{NH}), 7.92$ $(1 \mathrm{H}, \mathrm{d}, J=8.0 \mathrm{~Hz}, \mathrm{ArH}), 7.77(1 \mathrm{H}, \mathrm{d}, J=8.0 \mathrm{~Hz}, \mathrm{ArH}), 7.46-7.26(2 \mathrm{H}, \mathrm{m}, \mathrm{ArH}), 4.59$ (1H, m, $\mathrm{NCH}), 4.27(1 \mathrm{H}, \mathrm{m}, \mathrm{CH}), 4.10\left(1 \mathrm{H}, \mathrm{d}, J_{\mathrm{gem}}=14.9 \mathrm{~Hz}, \mathrm{SCH}\right), 4.00\left(1 \mathrm{H}, \mathrm{d}, J_{\text {gem }}=14.9 \mathrm{~Hz}, \mathrm{SCH}\right)$, $3.64\left(3 \mathrm{H}, \mathrm{s}, \mathrm{OCH}_{3}\right), 2.27-2.03(1 \mathrm{H}, \mathrm{bs}, \mathrm{OH}), 1.1\left(3 \mathrm{H}, \mathrm{d}, J=6.4 \mathrm{~Hz}, \mathrm{CH}_{3}\right)$. Anal. Calcd. for $\mathrm{C}_{14} \mathrm{H}_{16} \mathrm{~N}_{2} \mathrm{O}_{4} \mathrm{~S}_{2}$ (340.41): C, 49.39; H, 4.73; N, 8.22. Found C, 49.81; H, 4.78; N, 8.17. Mass spectrum, $m / z(\%): 340$ (23.5), 295 (50.7), 207 (36.7), 181 (48.8), 180 (49.1), 167 (100), 135 (25.7).

2-Benzothiazolylthioacetyl L-methionine methyl ester (2c). Colorless crystals $(0.83 \mathrm{~g}, 56.1 \%)$ mp 59- $61^{\circ} \mathrm{C}$, lit mp $62^{\circ} \mathrm{C} .{ }^{25}$

2-Benzothiazolylthioacetyl L-serine methyl ester (2d). Colorless crystals $(0.89 \mathrm{~g}, 68.3 \%) \mathrm{mp}$ 116- $118{ }^{\circ} \mathrm{C}$, lit. mp $115-116^{\circ} \mathrm{C} .{ }^{25}$

2-Benzothiazolylthioacetyl L-hydroxyproline methyl ester (3). Colorless crystals (0.64 g, $45.5 \%), \mathrm{R}_{\mathrm{f}}=0.25\left(\mathrm{~S}_{1}\right) \mathrm{mp} 104-106{ }^{\circ} \mathrm{C} .{ }^{1} \mathrm{H} \mathrm{NMR}\left(\mathrm{CDCl}_{3}\right): \delta=7.85(1 \mathrm{H}, \mathrm{d}, J=8.0 \mathrm{~Hz}, \mathrm{ArH}), 7.69$ $(1 \mathrm{H}, \mathrm{d}, J=8.0 \mathrm{~Hz}, \mathrm{ArH}), 7.41-7.22(2 \mathrm{H}, \mathrm{m}, \mathrm{ArH}), 4.59(1 \mathrm{H}, \mathrm{m}, \mathrm{NCH}), 4.37\left(1 \mathrm{H}, \mathrm{d}, J_{\mathrm{gem}}=15.0\right.$ $\mathrm{Hz}, \mathrm{SCH}), 4.19\left(1 \mathrm{H}, \mathrm{d}, J_{\text {gem }}=15.0 \mathrm{~Hz}, \mathrm{SCH}\right), 3.84\left(2 \mathrm{H}, \mathrm{d}, J=10.2 \mathrm{~Hz}, \mathrm{CH}_{2} \mathrm{~N}\right), 3.66(3 \mathrm{H}, \mathrm{s}$, $\left.\mathrm{OCH}_{3}\right)$, 3.36-2.80 (1H, bs, OH) 2.30-2.05 (2H, m, $\left.\mathrm{CH}_{2}\right)$. Anal. Calcd. for $\mathrm{C}_{15} \mathrm{H}_{16} \mathrm{~N}_{2} \mathrm{O}_{4} \mathrm{~S}_{2}$ (352.45): C, 51.12; H, 4.57; N, 7.94. Found: C, 51.20; H, 4.53; N, 8.04. MS:, m/z (\%): 352 (11.2), 208 (52.9), 180 (35.5), 167 (35.2), 144 (100), 136 (14.1).

\section{General procedure for preparation of compounds 4a-d, and 5}

To a solution of 2 or $3(10.0 \mathrm{mmol})$ in methanol $(30 \mathrm{ml})$, was added hydrazine hydrate $(3 \mathrm{ml}$, $48.0 \mathrm{mmol}$ ). The reaction mixture was refluxed for $8 \mathrm{hrs}$; after cooling to RT the precipitated hydrazide was filtered off, and washed with water and ethanol, followed by recrystallization from aqueous ethanol.

2-Benzothiazolylthioacetyl L-tyrosine hydrazide (4a). Colorless crystals $(0.36 \mathrm{~g}, 71.3 \%), \mathrm{R}_{\mathrm{f}}=$ $0.52\left(\mathrm{~S}_{2}\right), \mathrm{mp} 192-194{ }^{\circ} \mathrm{C} .{ }^{1} \mathrm{H}$ NMR $(200 \mathrm{MHz}, \mathrm{DMSO}): \delta 9.22(1 \mathrm{H}, \mathrm{s}, \mathrm{NH}), 8.62(1 \mathrm{H}, \mathrm{d}, J=8.5$ $\mathrm{Hz}, \mathrm{NH}), 8.02(1 \mathrm{H}, \mathrm{d}, J=7.6 \mathrm{~Hz}, \mathrm{ArH}), 7.84(1 \mathrm{H}, \mathrm{d}, J=7.6 \mathrm{~Hz}, \mathrm{ArH}), 7.50-7.32(2 \mathrm{H}, \mathrm{m}, \mathrm{ArH})$, $7.02(2 \mathrm{H}, \mathrm{d}, J=8.2 \mathrm{~Hz}, \mathrm{ArH}), 6.63(2 \mathrm{H}, \mathrm{d}, J=8.2 \mathrm{~Hz}, \mathrm{ArH}), 4.80(1 \mathrm{H}, \mathrm{bs}, \mathrm{OH}), 4.39(1 \mathrm{H}, \mathrm{m}$, $\mathrm{NCH}), 4.21\left(1 \mathrm{H}, \mathrm{d}, J_{\text {gem }}=14.0 \mathrm{~Hz}, \mathrm{SCH}\right), 4.11\left(1 \mathrm{H}, \mathrm{d}, J_{\text {gem }}=14.0 \mathrm{~Hz}, \mathrm{SCH}\right), 3.37\left(2 \mathrm{H}, \mathrm{s}, \mathrm{NH}_{2}\right)$, 2.88-2.65 (2H, m, $\mathrm{CH}_{2}$-Ar). IR (KBr): 3280 $\left(\mathrm{NH}_{2}\right),(\mathrm{NH}),(\mathrm{OH}), 1657(\mathrm{C}=\mathrm{O}$, amide), $1609(\mathrm{C}=\mathrm{O}$, amide). Anal. Calcd. For $\mathrm{C}_{18} \mathrm{H}_{18} \mathrm{~N}_{4} \mathrm{O}_{3} \mathrm{~S}_{2}$ (402.48): C, 53.71; H, 4.50; N, 13.92; Found C, 53.59; H, 4.51; N, 14.29. Mass spectrum, $m / z$ (\%): 402 (8.3), 371 (4.6), 225 (30), 208 (100), 180 (37.7), 167 (55.8), 136 (24.2), 107 (58.2).

2-Benzothiazolylthioacetyl DL-threonine hydrazide (4b). Colorless crystals (0.97 g, 79.5\%), $\mathrm{R}_{\mathrm{f}}=0.26\left(\mathrm{~S}_{1}\right) \mathrm{mp} 194-195{ }^{\circ} \mathrm{C} .{ }^{1} \mathrm{H}$ NMR $(200 \mathrm{MHz}, \mathrm{DMSO}): \delta=9.08(1 \mathrm{H}, \mathrm{s}, \mathrm{NH}), 8.28(1 \mathrm{H}, \mathrm{d}$, $J=8.3 \mathrm{~Hz}, \mathrm{NH}), 8.04(1 \mathrm{H}, \mathrm{d}, J=8.2 \mathrm{~Hz}, \mathrm{ArH}), 7.89(1 \mathrm{H}, \mathrm{d}, J=8.2 \mathrm{~Hz}, \mathrm{ArH}), 7.52-7.34(2 \mathrm{H}, \mathrm{m}$, ArH), 4.90 (1H, d, m, NCH), 4.27-4.03 (4H, m, SCH $\left.2, \mathrm{NH}_{2}\right), 1.01\left(3 \mathrm{H}, \mathrm{d}, J=5.9 \mathrm{~Hz}, \mathrm{CH}_{3}\right)$. IR $(\mathrm{KBr}): 3278\left(\mathrm{NH}_{2}\right),(\mathrm{NH}),(\mathrm{OH}), 1645\left(\mathrm{C}=\mathrm{O}\right.$, amide). Anal. Calcd. for $\mathrm{C}_{13} \mathrm{H}_{16} \mathrm{~N}_{4} \mathrm{O}_{3} \mathrm{~S}_{2}(340.45)$ : 
C, 45.86; H, 4.73; N, 16.45. Found C, 45.69; H, 4.73; N, 16.26. Mass spectrum, $\mathrm{m} / z$ (\%): 340(12.6), 309 (13.1), 281 (18.0), 207 (100), 180 (61.7), 167 (88.3), 136 (30.2).

2-Benzothiazolylthioacetyl L-methionine hydrazide (4c). Colorless crystals $(0.42 \mathrm{~g}, 84 \%), \mathrm{R}_{\mathrm{f}}$ $=0.27\left(\mathrm{~S}_{1}\right), \mathrm{mp} 175-177{ }^{\circ} \mathrm{C} .{ }^{1} \mathrm{H}$ NMR $(\mathrm{DMSO}): \delta=9.20(1 \mathrm{H}, \mathrm{s}, \mathrm{NH}), 8.54(1 \mathrm{H}, \mathrm{d}, J=8.2 \mathrm{~Hz}$, $\mathrm{NH}), 8.02(1 \mathrm{H}, \mathrm{d}, J=8.0 \mathrm{~Hz}, \mathrm{ArH}), 7.86(1 \mathrm{H}, \mathrm{d}, J=8.0 \mathrm{~Hz}, \mathrm{ArH}), 7.50-7.32$ (2H, m, ArH), 4.40$4.11\left(5 \mathrm{H}, \mathrm{m}, \mathrm{NCH}, \mathrm{SCH}_{2}, \mathrm{NH}_{2}\right), 2.49-2.36\left(2 \mathrm{H}, \mathrm{m}, \mathrm{SCH}_{2}\right), 1.98\left(3 \mathrm{H}, \mathrm{s}, \mathrm{SCH}_{3}\right), 1.91-1.83(2 \mathrm{H}$, m, $\left.\mathrm{CH}_{2}\right)$. IR ( $\left.\mathrm{KBr}\right): 3287\left(\mathrm{NH}_{2}, \mathrm{NH}\right), 1651(\mathrm{C}=\mathrm{O}$, amide), $1601(\mathrm{C}=\mathrm{O}$, amide). Anal. Calcd. for $\mathrm{C}_{14} \mathrm{H}_{18} \mathrm{~N}_{4} \mathrm{O}_{2} \mathrm{~S}_{3}$ (370.54): C, 45.38; H, 4.91; N, 15.12. Found: C, 45.62; H, 4.87; N, 14.81. Mass spectrum, $m / z \%): 370$ (30.4), 296 (18.4), 281 (34.1), 209 (26.5), 208 (100), 180 (72.7), 168 (38.8), 167 (75.8), 166 (16.1), 136 (26.1), 108 (20.8).

2-Benzothiazolylthioacetyl L-serine hydrazide (4d). Colorless crystals $(0.42 \mathrm{~g}, 83.8 \%), \mathrm{R}_{\mathrm{f}}=$ $0.16\left(\mathrm{~S}_{2}\right), \mathrm{mp} 208-210^{\circ} \mathrm{C}$. IR $(\mathrm{KBr}): 3268\left(\mathrm{NH}_{2}\right),(\mathrm{NH}),(\mathrm{OH}), 1644(\mathrm{C}=\mathrm{O}$, amide $), 1615(\mathrm{C}=\mathrm{O}$, amide). Anal. Calcd. for $\mathrm{C}_{12} \mathrm{H}_{14} \mathrm{~N}_{4} \mathrm{O}_{3} \mathrm{~S}_{2}$ (326.42): C, 44.15; H, 4.33; N, 17.15. Found: C, 44.22; H, 4.66; N, 16.98. Mass spectrum, $m / z, \%): 326$ (14.2), 295 (18.4), 209 (11.2), 208 (100), 180 (41.2), 168 (12.6), 167 (22.1), 136 (14.2), 108 (15.5).

2-Benzothiazolylthioacetyl L-hydroxyproline hydrazide (5). Colorless crystals (0.27 g, $54.6 \%), \mathrm{R}_{\mathrm{f}}=0.47\left(\mathrm{~S}_{2}\right), \mathrm{mp} 206-208{ }^{\circ} \mathrm{C} .{ }^{1} \mathrm{H}$ NMR (200 MHz, DMSO): $\delta=9.14(1 \mathrm{H}, \mathrm{s}, \mathrm{NH}), 8.01$ $(1 \mathrm{H}, \mathrm{d}, J=8.2 \mathrm{~Hz}, \mathrm{ArH}), 7.89(1 \mathrm{H}, \mathrm{d}, J=8.2 \mathrm{~Hz}, \mathrm{ArH}), 7.49-7.31(2 \mathrm{H}, \mathrm{m}, \mathrm{ArH}), 5.24(2 \mathrm{H}, \mathrm{s}$, $\left.\mathrm{NH}_{2}\right), 4.55-4.29\left(3 \mathrm{H}, \mathrm{m}, \mathrm{SCH}_{2}, \mathrm{NCH}\right), 3.78(1 \mathrm{H}, \mathrm{m}, \mathrm{OCH}), 3.60-3.51\left(2 \mathrm{H}, \mathrm{m}, \mathrm{CH}_{2}\right), 2.05-1.95$ $\left(2 \mathrm{H}, \mathrm{m}, \mathrm{CH}_{2}\right)$. IR $(\mathrm{KBr}): 3316\left(\mathrm{NH}_{2}\right),(\mathrm{NH}),(\mathrm{OH}), 1661(\mathrm{C}=\mathrm{O}$, amide $), 1625(\mathrm{C}=\mathrm{O}$, amide). Anal. Calcd. for $\mathrm{C}_{14} \mathrm{H}_{16} \mathrm{~N}_{4} \mathrm{O}_{3} \mathrm{~S}_{2}$ (352.56): C, 47.71; H, 4.57; N, 15.89. Found C, 47.57; H, 4.55; N, 16.18. Mass spectrum, $m / z, \%): 352$ (7.7), 208 (100), 180 (50.2), 167 (43.0), 144 (34.1), 136 (19.2).

\section{General procedure for preparation of dipeptide methyl esters (6a-g), and (7a,b)}

A cold solution $\left(-5^{\circ} \mathrm{C}\right)$ of the hydrazide 4 or $5(0.8 \mathrm{mmol})$ in acetic acid $(6 \mathrm{ml})$, hydrochloric acid $(5 \mathrm{~N}, 3 \mathrm{ml})$, and water $(25 \mathrm{ml})$, was treated portionwise under stirring with a cold solution $(0$ $\left.{ }^{\circ} \mathrm{C}\right)$ of sodium nitrite $(0.07 \mathrm{~g}, 1.0 \mathrm{mmol})$ in water $(3 \mathrm{ml})$. After stirring at the same temperature for $30 \mathrm{~min}$, the azide was extracted with cold ethyl acetate, and washed successively with cold water, $5 \% \mathrm{NaHCO}_{3}$, then water. After drying over anhydrous sodium sulphate, the dry cold azide solution was added dropwise to the amino acid ester [liberated from its hydrochloride $(0.9$ mmol) with triethylamine $(0.2 \mathrm{ml})$ in cold ethyl acetate $(30 \mathrm{ml})]$. Afterwards the mixture was kept $12 \mathrm{hrs}$ in the refrigerator and at RT for an additional $12 \mathrm{hrs}$. The reaction mixture was washed with $0.1 \mathrm{~N} \mathrm{HCl}$, water, $5 \% \mathrm{NaHCO}_{3}$, and water, then dried over anhydrous sodium sulphate. The solvent was evaporated in vacuum and the residue was crystallized from the ethyl acetate-petroleum to give the dipeptide methyl ester derivatives $\mathbf{6 a - g}$ and $\mathbf{7 a}, \mathbf{b}$.

2-Benzothiazolylthioacetyl L-methionyl L-methionine methyl ester (6a). Colorless crystals (0.25 g, 30.3\%), $\mathrm{R}_{\mathrm{f}}=0.74\left(\mathrm{~S}_{1}\right), \mathrm{mp} 119-122{ }^{\circ} \mathrm{C} .{ }^{1} \mathrm{H} \mathrm{NMR}\left(200 \mathrm{MHz}, \mathrm{CDCl}_{3}\right): \delta=8.26(1 \mathrm{H}, \mathrm{d}, J=$ $7.9 \mathrm{~Hz}, \mathrm{NH}), 7.89(1 \mathrm{H}, \mathrm{d}, J=7.9 \mathrm{~Hz}, \mathrm{ArH}), 7.78(1 \mathrm{H}, \mathrm{d}, J=7.9 \mathrm{~Hz}, \mathrm{ArH}), 7.48-7.32(2 \mathrm{H}, \mathrm{m}$, $\operatorname{ArH}), 6.95(1 \mathrm{H}, \mathrm{d}, J=7.9 \mathrm{~Hz}, \mathrm{NH}), 4.69-4.59(2 \mathrm{H}, \mathrm{m}, 2 \mathrm{NCH}), 4.07\left(1 \mathrm{H}, \mathrm{d}, J_{\mathrm{gem}}=14.6 \mathrm{~Hz}\right.$, 
$\mathrm{SCH}), 4.11\left(1 \mathrm{H}, \mathrm{d}, J_{\mathrm{gem}}=14.6 \mathrm{~Hz}, \mathrm{SCH}\right), 3.72\left(3 \mathrm{H}, \mathrm{s}, \mathrm{OCH}_{3}\right), 2.50-2.38\left(4 \mathrm{H}, \mathrm{m}, 2 \mathrm{CH}_{2} \mathrm{~S}\right), 2.02$ $\left(3 \mathrm{H}, \mathrm{s}, \mathrm{CH}_{3} \mathrm{~S}\right), 1.96\left(3 \mathrm{H}, \mathrm{s}, \mathrm{CH}_{3} \mathrm{~S}\right), 1.99-1.70\left(4 \mathrm{H}, \mathrm{m}, 2 \mathrm{CH}_{2}\right)$. Anal. Calcd. for $\mathrm{C}_{20} \mathrm{H}_{27} \mathrm{~N}_{3} \mathrm{O}_{4} \mathrm{~S}_{4}$ (501.17): C, 47.88; H, 5.42; N, 8.38. Found C, 47.53; H, 5.39; N, 8.57. Mass spectrum, $m / z$ (\%): 501 (3.1), 427 (23.4), 426 (21.3), 208 (71.5), 181 (8.9), 180 (33.2), 168 (25.1), 167 (40.7), 166 (30.9), 136 (14.2).

2-Benzothiazolylthioacetyl L-methionyl L-serine methyl ester (6b). Colorless crystals $(0.23 \mathrm{~g}$, $31.7 \%), \mathrm{R}_{\mathrm{f}}=0.28\left(\mathrm{~S}_{1}\right), \mathrm{mp} 127-130{ }^{\circ} \mathrm{C} .{ }^{1} \mathrm{H} \mathrm{NMR}\left(200 \mathrm{MHz}, \mathrm{CDCl}_{3}\right): \delta=8.37(1 \mathrm{H}, \mathrm{d}, J=7.0 \mathrm{~Hz}$, $\mathrm{NH}), 7.97(1 \mathrm{H}, \mathrm{d}, J=7.4 \mathrm{~Hz}, \mathrm{ArH}), 7.82(1 \mathrm{H}, \mathrm{d}, J=7.4 \mathrm{~Hz}, \mathrm{ArH}), 7.53-7.35$ (3H, m, 2ArH, NH), 4.73-4.63 (2H, m, 2NCH), 4.15-3.87 (4H, m, $\left.\mathrm{CH}_{2} \mathrm{O}, \mathrm{CH}_{2} \mathrm{~S}\right), 3.79\left(3 \mathrm{H}, \mathrm{s}, \mathrm{OCH}_{3}\right), 2.54(2 \mathrm{H}, \mathrm{m}$, $\left.\mathrm{CH}_{2} \mathrm{~S}\right), 2.19-2.01\left(2 \mathrm{H}, \mathrm{m}, \mathrm{CH}_{2}\right), 1.98\left(3 \mathrm{H}, \mathrm{s}, \mathrm{CH}_{3} \mathrm{~S}\right)$. Anal. Calcd. for $\mathrm{C}_{18} \mathrm{H}_{23} \mathrm{~N}_{3} \mathrm{O}_{5} \mathrm{~S}_{3}$ (457.62): C, 47.25; H, 5.07; N, 9.18. Found C, 47.24; H, 5.00; N, 9.41. Mass spectrum, $m / z$ (\%): 457 (6.7), 382 (15.2), 264 (11.4), 225 (16.5), 209 (14.3), 208 (75.6), 181 (40.2), 180 (100), 168 (30.1), 167 (43.4), 166 (37.3), 136 (19.1), 108 (10.9).

2-Benzothiazolylthioacetyl L-serinyl L-methionine methyl ester (6c). Colorless crystals $(0.27$ $\mathrm{g}, 37.3 \%), \mathrm{R}_{\mathrm{f}}=0.45\left(\mathrm{~S}_{1}\right), \mathrm{mp} 101-104{ }^{\circ} \mathrm{C} .{ }^{1} \mathrm{H} \mathrm{NMR}\left(200 \mathrm{MHz}, \mathrm{CDCl}_{3}\right): \delta=8.58(1 \mathrm{H}, \mathrm{d}, J=7.2$ $\mathrm{Hz}, \mathrm{NH}), 7.95(1 \mathrm{H}, \mathrm{d}, J=7.8 \mathrm{~Hz}, \mathrm{ArH}), 7.76(1 \mathrm{H}, \mathrm{d}, J=7.8 \mathrm{~Hz}, \mathrm{ArH}), 7.42-7.26(3 \mathrm{H}, \mathrm{m}, 2 \mathrm{ArH}$, $\mathrm{NH}), 4.60-4.54(2 \mathrm{H}, \mathrm{m}, 2 \mathrm{NCH}), 4.10\left(1 \mathrm{H}, \mathrm{d}, J_{\mathrm{gem}}=14.4 \mathrm{~Hz}, \mathrm{SCH}\right), 3.95\left(1 \mathrm{H}, \mathrm{d}, J_{\mathrm{gem}}=14.4 \mathrm{~Hz}\right.$, $\mathrm{SCH}), 3.70\left(3 \mathrm{H}, \mathrm{s}, \mathrm{OCH}_{3}\right), 3.62\left(2 \mathrm{H}, \mathrm{m}, \mathrm{CH}_{2} \mathrm{O}\right), 2.41-2.34\left(2 \mathrm{H}, \mathrm{m}, \mathrm{CH}_{2} \mathrm{~S}\right), 2.10-2.01(2 \mathrm{H}, \mathrm{m}$, $\mathrm{CH}_{2}$ ), 1.97 (3H, s, $\mathrm{CH}_{3} \mathrm{~S}$ ). Anal. Calcd. for $\mathrm{C}_{18} \mathrm{H}_{23} \mathrm{~N}_{3} \mathrm{O}_{5} \mathrm{~S}_{3}$ (457.62): C, 47.25; H, 5.07; N, 9.18. Found, C, 47.36; H, 4.94; N, 9.35. Mass spectrum, $m / z$ (\%): 457 (5.1), 383 (40.0), 382 (31.1), 225 (30.5), 209 (13.4), 208 (100), 181 (20.4), 180 (51.0), 168 (29.9), 167 (43.4), 166 (27.8), 136 (21.0).

2-Benzothiazolylthioacetyl L-serinyl L-serine methyl ester (6d). Colorless crystals (0.22 g, $33.7 \%) \mathrm{R}_{\mathrm{f}}=0.23\left(\mathrm{~S}_{1}\right), \mathrm{mp} 183-185{ }^{\circ} \mathrm{C} .{ }^{1} \mathrm{H} \mathrm{NMR}(200 \mathrm{MHz}, \mathrm{DMSO}): \delta=8.53(1 \mathrm{H}, \mathrm{d}, J=7.5 \mathrm{~Hz}$, $\mathrm{NH}), 8.25(1 \mathrm{H}, \mathrm{d}, J=6.4 \mathrm{~Hz}, \mathrm{NH}), 8.03(1 \mathrm{H}, \mathrm{d}, J=7.0 \mathrm{~Hz}, \mathrm{ArH}), 7.87(1 \mathrm{H}, \mathrm{d}, J=7.3 \mathrm{~Hz}, \mathrm{ArH})$, 7.51-7.33 (2H, m, ArH), 5.08-4.96 (2H, m, NCH), 4.48-4.41 (2H, m, $\left.\mathrm{CH}_{2} \mathrm{~S}\right), 4.25\left(3 \mathrm{H}, \mathrm{s}, \mathrm{OCH}_{3}\right)$, 3.51-2.99 (4H, m, 2 $\mathrm{CH}_{2} \mathrm{O}$ ). Anal. Calcd. for $\mathrm{C}_{16} \mathrm{H}_{19} \mathrm{~N}_{3} \mathrm{O}_{6} \mathrm{~S}_{2}$ (413.5): C, 46.47; H, 4.63; N, 10.16; Found C, 46.30; H, 4.63; N, 10.67. 6d Mass spectrum, $m / z(\%): 414$ (5.6), 413 (4.6), 383 (10.2), 382 (7.4), 250 (11.1), 249 (12.0), 210 (25.0), 209 (16.7), 208 (69.4), 181 (36.1), 180 (40.7), 168 (26.9), 167 (100), 166 (21.3), 136 (26.9), 109 (26.9), 108 (38.0).

2-Benzothiazolylthioacetyl L-tyrosyl L-methionine methyl ester (6e). Colorless crystals $(0.30$ $\mathrm{g}, 34.1 \%), \mathrm{R}_{\mathrm{f}}=0.12\left(\mathrm{~S}_{1}\right), \mathrm{mp} 97-100{ }^{\circ} \mathrm{C} .{ }^{1} \mathrm{H} \mathrm{NMR}\left(200 \mathrm{MHz}, \mathrm{CDCl}_{3}\right): \delta=8.18(1 \mathrm{H}, \mathrm{d}, J=7.8$ $\mathrm{Hz}, \mathrm{NH}), 7.80-7.72$ (2H, m, ArH), 7.46-7.23 (3H, m, 2ArH, NH), 6.85 (2H, d, J=8.1 Hz, ArH), $6.52(2 \mathrm{H}, \mathrm{d}, J=8.1 \mathrm{~Hz}, \mathrm{ArH}), 4.75-4.55(2 \mathrm{H}, \mathrm{m}, 2 \mathrm{NCH}), 4.05\left(1 \mathrm{H}, \mathrm{d}, J_{\text {gem }}=14.5 \mathrm{~Hz}, \mathrm{SCH}\right), 3.87$ $\left(1 \mathrm{H}, \mathrm{d}, J_{\mathrm{gem}}=14.5 \mathrm{~Hz}, \mathrm{SCH}\right), 3.69\left(3 \mathrm{H}, \mathrm{s}, \mathrm{OCH}_{3}\right), 2.96-2.91\left(2 \mathrm{H}, \mathrm{m}, \mathrm{CH}_{2}\right), 2.36\left(2 \mathrm{H}, \mathrm{m}, \mathrm{SCH}_{2}\right)$, $1.98\left(3 \mathrm{H}, \mathrm{s}, \mathrm{SCH}_{3}\right), 1.89-1.75\left(2 \mathrm{H}, \mathrm{m}, \mathrm{CH}_{2}\right)$. Anal. Calcd. for $\mathrm{C}_{24} \mathrm{H}_{27} \mathrm{~N}_{3} \mathrm{O}_{5} \mathrm{~S}_{3}$ (533.67): C, 54.01; H, 5.09; N, 7.87. Found C, 53.70; H, 5.18; N, 7.90. Mass spectrum, $m / z$ (\%): 533 (4.2), 225 (69.1), 208 (100), 180 (35.2), 167 (28.7), 147 (21.7), 136 (23.1), 107 (43.7).

2-Benzothiazolylthioacetyl L-tyrosyl L-serine methyl ester (6f). Colorless crystals (0.23 g, $30.0 \%), \mathrm{R}_{\mathrm{f}}=0.41\left(\mathrm{~S}_{2}\right), \mathrm{mp} 157-159{ }^{\circ} \mathrm{C} .{ }^{1} \mathrm{H}$ NMR (200 MHz, DMSO): $\delta=9.21(1 \mathrm{H}, \mathrm{brs}, \mathrm{NH})$, 
$8.54(1 \mathrm{H}, \mathrm{d}, J=7.9 \mathrm{~Hz}, \mathrm{NH}), 8.02(2 \mathrm{H}, \mathrm{d}, J=7.9 \mathrm{~Hz}, \mathrm{ArH}), 7.82(2 \mathrm{H}, \mathrm{d}, J=7.9 \mathrm{~Hz}, \mathrm{ArH}), 7.50-$ $7.32(2 \mathrm{H}, \mathrm{m}, \mathrm{ArH}), 7.06(2 \mathrm{H}, \mathrm{d}, J=8.3 \mathrm{~Hz}, \mathrm{ArH}), 6.62(2 \mathrm{H}, \mathrm{d}, J=8.3 \mathrm{~Hz}, \mathrm{ArH}), 5.10(1 \mathrm{H}, \mathrm{m}$, $\mathrm{NCH}), 4.59(1 \mathrm{H}, \mathrm{m}, \mathrm{NCH}), 4.19\left(1 \mathrm{H}, \mathrm{d}, J_{\text {gem }}=14.8 \mathrm{~Hz}, \mathrm{SCH}\right), 4.09\left(1 \mathrm{H}, \mathrm{d}, J_{\text {gem }}=14.8 \mathrm{~Hz}\right.$, $\mathrm{SCH}), 3.36\left(3 \mathrm{H}, \mathrm{s}, \mathrm{OCH}_{3}\right), 2.91-2.65\left(4 \mathrm{H}, \mathrm{m}, 2 \mathrm{CH}_{2}\right)$. Anal. Calcd. for $\mathrm{C}_{22} \mathrm{H}_{23} \mathrm{~N}_{3} \mathrm{O}_{6} \mathrm{~S}_{2}$ (489.56): C, 53.97; H, 4.73; N, 8.58. Found C, 53.63; H, 4.86; N, 8.66. Mass spectrum, $m / z(\%)$ : 489 (9.3), 470 (25.8), 225 (48.4), 224 (38.7), 208 (100), 207 (87.1), 179 (67.7), 169 (48.4), 167 (77.4), 166 (93.5), 147 (29.0), 135 (87.1), 107 (77.4), 106 (67.7).

2-Benzothiazolylthioacetyl DL-threonyl L-methionine methyl ester (6g). Colorless crystals $(0.29 \mathrm{~g}, 43.3 \%), \mathrm{R}_{\mathrm{f}}=0.58\left(\mathrm{~S}_{1}\right), \mathrm{mp} 135-137{ }^{\circ} \mathrm{C} .{ }^{1} \mathrm{H} \mathrm{NMR}\left(300 \mathrm{MHz}, \mathrm{CDCl}_{3}\right): \delta=8.59(1 \mathrm{H}, \mathrm{d}, J=$ $8.4 \mathrm{~Hz}, \mathrm{NH}), 7.90(1 \mathrm{H}, \mathrm{d}, J=7.8 \mathrm{~Hz}, \mathrm{ArH}), 7.71(1 \mathrm{H}, \mathrm{d}, J=7.8 \mathrm{~Hz}, \mathrm{ArH}), 7.40-7.12(3 \mathrm{H}, \mathrm{m}$, 2ArH, NH), $5.84(1 \mathrm{H}, \mathrm{m}, \mathrm{NCH}), 4.76(1 \mathrm{H}, \mathrm{m}, \mathrm{NCH}), 4.52(1 \mathrm{H}, \mathrm{m}, \mathrm{OCH}), 4.33\left(2 \mathrm{H}, \mathrm{m}, \mathrm{SCH}_{2}\right)$, $3.92\left(2 \mathrm{H}, \mathrm{m}, \mathrm{SCH}_{2}\right), 3.66\left(3 \mathrm{H}, \mathrm{s}, \mathrm{OCH}_{3}\right), 2.40\left(2 \mathrm{H}, \mathrm{m}, \mathrm{CH}_{2}\right), 1.98\left(3 \mathrm{H}, \mathrm{s}, \mathrm{SCH}_{3}\right) 1.04(3 \mathrm{H}, \mathrm{d}, J=$ $6.4 \mathrm{~Hz}, \mathrm{CH}_{3}$ ). Anal. Calcd. for $\mathrm{C}_{19} \mathrm{H}_{25} \mathrm{~N}_{3} \mathrm{O}_{5} \mathrm{~S}_{3}$ (471.09): C, 48.38; H, 5.34; N, 8.91; Found C, 48.42; H, 5.15; N, 9.27. Mass spectrum, $m / z(\%): 471$ (6.6), 470 (7.5), 397 (28.9), 396 (20.4), 225 (59.0), 224 (48.3), 208 (100), 207 (88.6), 180 (46.9), 179 (39.7), 168 (43.6), 167 (67.3), 166 (53.5), 136 (21.8).

2-Benzothiazolylthioacetyl L-hydroxyprolyl L-methionine methyl ester (7a). Colorless crystals $(0.30 \mathrm{~g}, 39.1 \%), \mathrm{R}_{\mathrm{f}}=0.22\left(\mathrm{~S}_{1}\right), \mathrm{mp} 118-120{ }^{\circ} \mathrm{C} .{ }^{1} \mathrm{H}$ NMR $\left(200 \mathrm{MHz}, \mathrm{CDCl}_{3}\right): \delta=$ 7.81(1H, d, $J=7.7 \mathrm{~Hz}, \mathrm{ArH}), 7.72(1 \mathrm{H}, \mathrm{d}, J=7.7 \mathrm{~Hz}, \mathrm{ArH}), 7.40-7.26$ (3H, m, 2ArH, NH), 4.64$4.40(3 \mathrm{H}, \mathrm{m}, 2 \mathrm{NCH}, \mathrm{OCH}), 4.28\left(1 \mathrm{H}, \mathrm{d}, J_{\mathrm{gem}}=15.2 \mathrm{~Hz}, \mathrm{SCH}\right), 4.18\left(1 \mathrm{H}, \mathrm{d}, J_{\mathrm{gem}}=15.2 \mathrm{~Hz}\right.$, $\mathrm{SCH}), 3.84\left(2 \mathrm{H}, \mathrm{m}, \mathrm{CH}_{2} \mathrm{~N}\right), 3.68\left(3 \mathrm{H}, \mathrm{s}, \mathrm{OCH}_{3}\right), 2.42\left(2 \mathrm{H}, \mathrm{m}, \mathrm{CH}_{2} \mathrm{~S}\right), 2.14\left(2 \mathrm{H}, \mathrm{m}, \mathrm{CH}_{2}\right), 1.98$ $\left(3 \mathrm{H}, \mathrm{s}, \mathrm{SCH}_{3}\right), 1.92\left(2 \mathrm{H}, \mathrm{m}, \mathrm{CH}_{2}\right)$. Anal. Calcd. for $\mathrm{C}_{20} \mathrm{H}_{25} \mathrm{~N}_{3} \mathrm{O}_{5} \mathrm{~S}_{3}(483.61)$ : C, 49.67; H, 5.21; N, 8.68. Found C, 49.83; H, 5.21; N, 8.60. Mass spectrum, $m / z$ (\%): 484 (10.4), 482 (14.9), 409 (35.8), 408 (28.4), 209 (25.4), 208 (100), 207 (79.1), 180 (49.3), 179 (25.4), 167 (59.7), 166 (53.7), 135 (20.9), 134 (25.4), 128 (32.8).

2-Benzothiazolylthioacetyl L-hydroxyprolyl L-serine methyl ester (7b). Colorless crystals $(0.29 \mathrm{~g}, 41.5 \%), \mathrm{R}_{\mathrm{f}}=0.08\left(\mathrm{~S}_{1}\right), \mathrm{mp} 198-200{ }^{\circ} \mathrm{C} .{ }^{1} \mathrm{H}$ NMR $(200 \mathrm{MHz}, \mathrm{DMSO}): \delta=8.34(1 \mathrm{H}, \mathrm{d}$, $J=5.8 \mathrm{~Hz}, \mathrm{NH}), 8.01(1 \mathrm{H}, \mathrm{d}, J=7.7 \mathrm{~Hz}, \mathrm{ArH}), 7.89(1 \mathrm{H}, \mathrm{d}, J=7.7 \mathrm{~Hz}, \mathrm{ArH}), 7.52-7.33(2 \mathrm{H}, \mathrm{m}$, $\mathrm{ArH}), 5.22-5.04(2 \mathrm{H}, \mathrm{m}, 2 \mathrm{NCH}), 4.55-4.29\left(4 \mathrm{H}, \mathrm{m}, \mathrm{SCH}_{2}, \mathrm{OCH}_{2}\right), 4.07(1 \mathrm{H}, \mathrm{m}, \mathrm{OCH}), 3.98$ $\left(2 \mathrm{H}, \mathrm{m}, \mathrm{NCH}_{2}\right), 3.62\left(3 \mathrm{H}, \mathrm{s}, \mathrm{OCH}_{3}\right), 2.01-1.98\left(2 \mathrm{H}, \mathrm{m}, \mathrm{CH}_{2}\right)$. Anal. Calcd. for $\mathrm{C}_{18} \mathrm{H}_{21} \mathrm{~N}_{3} \mathrm{O}_{6} \mathrm{~S}_{2}$ (439.5): C, 49.19; H, 4.81; N, 9.56: Found C, 49.36; H, 4.83; N, 9.52. Mass spectrum, $m / z$ (\%): 439 (4.5), 438 (7.3), 209 (21.9), 208 (100), 207 (87.6), 180 (45.5), 179 (30.9), 167 (29.8), 166 (27.5), 136 (14.6), 135 (13.5).

2-Benzothiazolylthioacetyl $\boldsymbol{O}$-acetyl L-hydroxyproline methyl ester (8). A mixture of 3 (1.0 $\mathrm{g}, 2.85 \mathrm{mmol})$, acetic anhydride $(2.0 \mathrm{ml})$ in pyridine $(2.0 \mathrm{ml})$ was stirred at $0{ }^{\circ} \mathrm{C}$ for $1 \mathrm{hr}$. and at room temperature overnight. The reaction mixture was poured into ice, and the solid precipitate was filtered, washed with water and dried in air. Crystallization from ethyl acetate / petroleum gave colorless crystals of $\mathbf{8}(0.41 \mathrm{~g}, 36.6 \%), \mathrm{R}_{\mathrm{f}}=0.81\left(\mathrm{~S}_{1}\right) \mathrm{mp} 59-60{ }^{\circ} \mathrm{C} .{ }^{1} \mathrm{H} \mathrm{NMR}\left(\mathrm{CDCl}_{3}\right): \delta=$ $7.85(1 \mathrm{H}, \mathrm{d}, J=8.0 \mathrm{~Hz}, \mathrm{ArH}), 7.76(1 \mathrm{H}, \mathrm{d}, J=8.0 \mathrm{~Hz}, \mathrm{ArH}), 7.43-7.20(2 \mathrm{H}, \mathrm{m}, \mathrm{ArH}), 5.39-5.21$ $(1 \mathrm{H}, \mathrm{m}, \mathrm{CH}) 4.59(1 \mathrm{H}, \mathrm{t}, J=8.2 \mathrm{~Hz}, \mathrm{NCH}), 4.43\left(1 \mathrm{H}, \mathrm{d}, J_{\text {gem }}=14.8 \mathrm{~Hz}, \mathrm{SCH}\right), 4.22\left(1 \mathrm{H}, \mathrm{d}, J_{\text {gem }}=\right.$ 
$14.8 \mathrm{~Hz}, \mathrm{SCH}), 4.07-3.81\left(2 \mathrm{H}, \mathrm{m}, \mathrm{CH}_{2} \mathrm{~N}\right), 3.69\left(3 \mathrm{H}, \mathrm{s}, \mathrm{OCH}_{3}\right), 2.40-2.15\left(2 \mathrm{H}, \mathrm{m}, \mathrm{CH}_{2}\right), 2.02$ (3H, s, $\mathrm{CH}_{3}$ ). Anal. Calcd. for $\mathrm{C}_{17} \mathrm{H}_{18} \mathrm{~N}_{2} \mathrm{O}_{5} \mathrm{~S}_{2}$ (394.49): C, 51.76; H, 4.59; N, 7.10. Found C, 52.08; H, 4.75; N, 6.95. Mass spectrum, m/z (\%): 394 (6.6), 335 (2.1), 209 (12.4), 208 (100), 207 (9.0), 186 (39.7), 180 (46.1), 179 (6.9), 167 (32.5), 166 (9.5), 136 (18.8), 126 (65.1), 108 (18.7).

\section{General procedure for preparation of compounds 10a, b}

To a solution of compound $\mathbf{2 d}$ or $\mathbf{3}(1.5 \mathrm{mmol})$ and compound $\mathbf{9}^{26}$ (1.5 mmol) in dry dichloromethane $(25 \mathrm{ml})$, was added trimethylsilyl trifluoromethanesulfonate (TMSOTF) $(30 \mu 1$, $0.15 \mathrm{mmol}$ ) under stirring. After $1.5 \mathrm{hr}$. the reaction mixture was neutralized with solid sodium bicarbonate, filtered and concentrated in vacuum. The residue was purified by preparative TLC using solvent system $\left(\mathrm{S}_{3}\right)$.

2-Benzothiazolylthioacetyl $\boldsymbol{O}$-(2:3,5:6-di- $\boldsymbol{O}$-isopropylidene- $\alpha$-D-mannofuranosyl) L-serine methyl ester (10a). Pale yellow oil $(0.31 \mathrm{~g}, 35.8 \%), \mathrm{R}_{\mathrm{f}}=0.76\left(\mathrm{~S}_{3}\right) .{ }^{1} \mathrm{H} \mathrm{NMR}\left(200 \mathrm{MHz}, \mathrm{CDCl}_{3}\right)$ : $\delta=8.61(1 \mathrm{H}, \mathrm{d}, J=8.2 \mathrm{~Hz}, \mathrm{NH}), 7.92(1 \mathrm{H}, \mathrm{d}, J=8.0 \mathrm{~Hz}, \mathrm{ArH}), 7.77(1 \mathrm{H}, \mathrm{d}, J=8.0 \mathrm{~Hz}, \operatorname{ArH})$, 7.47-7.26 (2H, m, ArH), $5.34(1 \mathrm{H}, \mathrm{s}, 1-\mathrm{H}), 4.78-4.71(2 \mathrm{H}, \mathrm{m}, 3-\mathrm{H}, \mathrm{NCH}), 4.59(1 \mathrm{H}, \mathrm{d}, J=5.8$ $\mathrm{Hz}, 2-\mathrm{H}), 4.39$ (1H, m, 5-H), 4.23-3.97 (5H, m, 4-H, $\left.\mathrm{CH}_{2} \mathrm{O}, \mathrm{SCH}_{2}\right), 3.92-3.63$ (2H, m, 6-H, 6$\mathrm{H}), 3.68\left(3 \mathrm{H}, \mathrm{s}, \mathrm{OCH}_{3}\right), 1.41\left(3 \mathrm{H}, \mathrm{s}, \mathrm{CH}_{3}\right), 1.33\left(3 \mathrm{H}, \mathrm{s}, \mathrm{CH}_{3}\right), 1.29\left(3 \mathrm{H}, \mathrm{s}, \mathrm{CH}_{3}\right), 1.22(3 \mathrm{H}, \mathrm{s}$, $\mathrm{CH}_{3}$ ). Anal. Calcd. for $\mathrm{C}_{25} \mathrm{H}_{32} \mathrm{~N}_{2} \mathrm{O}_{9} \mathrm{~S}_{2}$ (568.66): C, 52.80; H, 5.67; N, 4.93. Found C, 52.95; H, 5.66; N, 4.85. Mass spectrum, $m / z$ (\%): 568 (2.1), 567 (8.6), 310 (23.3), 295 (39.0), 208 (100), 181 (33.4), 180 (49.0), 168 (27.9), 167 (45.1), 136 (21.5), 108 (11.2), 101 (19.4).

\section{2-Benzothiazolylthioacetyl $\boldsymbol{O}$-(2:3,5:6-di- $\boldsymbol{O}$-isopropylidene- $\alpha$-D-mannofuranosyl) L-} hydroxyproline methyl ester (10b). Pale yellow oil $(0.24 \mathrm{~g}, 28.0 \%), \mathrm{R}_{\mathrm{f}}=0.82\left(\mathrm{~S}_{3}\right) .{ }^{1} \mathrm{H} \mathrm{NMR}$ $\left(200 \mathrm{MHz}, \mathrm{CDCl}_{3}\right): \delta=7.86-7.09(4 \mathrm{H}, \mathrm{m}, \mathrm{ArH}), 5.09(1 \mathrm{H}, \mathrm{s}, 1-\mathrm{H}), 4.79(1 \mathrm{H}, \mathrm{m}, 3-\mathrm{H}), 4.67-4.51$ $(2 \mathrm{H}, \mathrm{m}, 2-\mathrm{H}, \mathrm{NCH}), 4.47-4.14$ (5H, m, $\left.\mathrm{OCH}, 5-\mathrm{H}, 4-\mathrm{H}, \mathrm{SCH}_{2}\right), 4.12-3.80\left(4 \mathrm{H}, \mathrm{m}, \mathrm{NCH}_{2}, 6-\mathrm{H}\right.$, 6- $\mathrm{H}), 3.69$ (3H, s, $\left.\mathrm{OCH}_{3}\right), 2.34-2.05\left(2 \mathrm{H}, \mathrm{m}, \mathrm{CH}_{2}\right), 1.45\left(3 \mathrm{H}, \mathrm{s}, \mathrm{CH}_{3}\right), 1.37\left(3 \mathrm{H}, \mathrm{s}, \mathrm{CH}_{3}\right), 1.31$ $\left(3 \mathrm{H}, \mathrm{s}, \mathrm{CH}_{3}\right), 1.25$ (3H, s, $\left.\mathrm{CH}_{3}\right)$. Anal. Calcd. for $\mathrm{C}_{27} \mathrm{H}_{34} \mathrm{~N}_{2} \mathrm{O}_{9} \mathrm{~S}_{2}(594.71)$ : C, 54.53; H, 5.76; N, 4.71; Found C, 54.59; H, 5.77; N, 4.75. Mass spectrum, $m / z$ (\%): 594 (1.2), 335 (25.0), 276 (13.6), 209 (10.1), 208 (100), 180 (55.3), 168 (29.4), 167 (49.4), 136 (14.1), 127 (16.5), 108 (10.9), 101 (11.6).

2-Benzothiazolylthioacetyl L-methionyl hydrazinocarbonylacetic acid ethyl ester (12). A mixture of hydrazide $4 \mathbf{c}(0.30 \mathrm{~g}, 1.0 \mathrm{mmol})$ and diethyl malonate $(5 \mathrm{ml})$ was heated at $150^{\circ} \mathrm{C}$ for $8 \mathrm{~h}$. The reaction mixture was poured into ether, the solid precipitate filtered and washed with ether. Crystallization from ethanol- water gave pale yellow crystals $(0.12 \mathrm{~g}, 30.7 \%), \mathrm{R}_{\mathrm{f}}=0.78$ $\left(\mathrm{S}_{1}\right), \mathrm{mp} 156-158{ }^{\circ} \mathrm{C} .{ }^{1} \mathrm{H}$ NMR $\left(\mathrm{CDCl}_{3}\right): \delta=9.70(2 \mathrm{H}, \mathrm{s}, 2 \mathrm{NH}), 8.20(1 \mathrm{H}, \mathrm{d}, J=8.2 \mathrm{~Hz}, \mathrm{NH})$, $7.82(1 \mathrm{H}, \mathrm{d}, J=8.0 \mathrm{~Hz}, \mathrm{ArH}), 7.68(1 \mathrm{H}, \mathrm{d}, J=8.0 \mathrm{~Hz}, \mathrm{ArH}), 7.36-7.18(2 \mathrm{H}, \mathrm{m}, \mathrm{ArH}), 4.68-4.59$ $(1 \mathrm{H}, \mathrm{m}, \mathrm{NCH}), 4.12-4.06\left(2 \mathrm{H}, \mathrm{m}, \mathrm{CH}_{2} \mathrm{O}\right), 4.02\left(1 \mathrm{H}, \mathrm{d}, J_{\mathrm{gem}}=13.8 \mathrm{~Hz}, \mathrm{SCH}\right), 3.88\left(1 \mathrm{H}, \mathrm{d}, J_{\text {gem }}=\right.$ $13.8 \mathrm{~Hz}, \mathrm{SCH}), 3.25\left(2 \mathrm{H}, \mathrm{s}, \mathrm{CH}_{2}\right), 2.40-2.32\left(2 \mathrm{H}, \mathrm{m}, \mathrm{CH}_{2} \mathrm{~S}\right), 2.07-1.93\left(2 \mathrm{H}, \mathrm{m}, \mathrm{CH}_{2}\right), 1.82(3 \mathrm{H}$, s, $\left.\mathrm{CH}_{3} \mathrm{~S}\right), 1.16\left(3 \mathrm{H}, \mathrm{t}, J=5.6 \mathrm{~Hz}, \mathrm{CH}_{3}\right)$. Anal. Calcd. for $\mathrm{C}_{19} \mathrm{H}_{24} \mathrm{~N}_{4} \mathrm{O}_{5} \mathrm{~S}_{3}$ (484.09): C, 47.09; $\mathrm{H}$, 4.99; N, 11.56. Found: C, 47.35; H, 4.61; N, 11.44. Mass spectrum, $m / z(\%): 484(3.1), 208$ (35.5), 207 (12.2), 180 (20.5), 168 (21.0), 167 (100), 136 (10.1), 135 (10.4), 134 (7.0). 
General procedure for preparation of compounds 13a, b, 14a-f and 15

A mixture of $\mathbf{4 c}$ or $\mathbf{4 d}(1.0 \mathrm{mmol})$ and carbonyl compound $(1.0 \mathrm{mmol}$, or $0.5 \mathrm{mmol}$ in the case of terephthalaldehyde) was heated under reflux in ethanol $(20 \mathrm{ml})$ for 10 hours. After cooling to room temperature the resulting solid was filtered, washed with cold ethanol, and recrystallized from aqueous ethanol.

3-[2-Benzothiazolylthioacetyl L-methionyl]-1,3-dihydroindol-2-one hydrazone (13a). Yellow crystals $(0.81 \mathrm{~g}, 59.3 \%) \mathrm{R}_{\mathrm{f}}=0.52\left(\mathrm{~S}_{1}\right), \mathrm{mp} 137-139{ }^{\circ} \mathrm{C} .{ }^{1} \mathrm{H}$ NMR (DMSO): $\delta=13.39(1 \mathrm{H}, \mathrm{s}, \mathrm{NH}$ isatin $\left.{ }^{27}\right), 11.24(1 \mathrm{H}, \mathrm{s}, \mathrm{NH}), 8.95(1 \mathrm{H}, \mathrm{m}, \mathrm{NH}), 7.97-7.80(2 \mathrm{H}, \mathrm{m}, \mathrm{ArH}), 7.52-7.25(4 \mathrm{H}, \mathrm{m}$, $\mathrm{ArH}), 7.08-6.93(2 \mathrm{H}, \mathrm{m}, \mathrm{ArH}), 5.43(1 \mathrm{H}, \mathrm{m}, \mathrm{NCH}), 4.55-4.23\left(2 \mathrm{H}, \mathrm{m}, \mathrm{CH}_{2} \mathrm{~S}\right), 2.50(2 \mathrm{H}, \mathrm{m}$, $\mathrm{CH}_{2} \mathrm{~S}$ ), 2.01 (3H, s, $\left.\mathrm{CH}_{3} \mathrm{~S}\right), 2.20-1.83\left(2 \mathrm{H}, \mathrm{m}, \mathrm{CH}_{2}\right)$. Anal. Calcd. for $\mathrm{C}_{22} \mathrm{H}_{21} \mathrm{~N}_{5} \mathrm{O}_{3} \mathrm{~S}_{3}$ (499.66): C, 52.89; H, 4.24; N, 14.02. Found C, 52.47; H, 4.19; N, 13.77\%. Mass spectrum, $m / z(\%): 499$ (6.8), 424 (11.7), 355 (5.8), 339 (6.8), 291 (6.8), 208 (100), 207 (78.6), 180 (39.8), 179 (27.3), 168 (35.9), 167 (79.6), 166 (44.7), 160 (21.4), 145 (15.5), 136 (21.4), 132 (17.5), 118 (23.3), 108 (25.2), 91 (26.2), 90 (32.0).

3-[2-Benzothiazolylthioacetyl L-serinyl]-1,3-dihydroindol-2-one hydrazone (13b). Yellow crystals $(0.73 \mathrm{~g}, 52.5 \%) \mathrm{R}_{\mathrm{f}}=0.27\left(\mathrm{~S}_{1}\right), \mathrm{mp} 175-177{ }^{\circ} \mathrm{C} .{ }^{1} \mathrm{H}$ NMR (DMSO): $\delta=13.37(1 \mathrm{H}, \mathrm{s}, \mathrm{NH}$ isatin $\left.^{27}\right), 11.24(1 \mathrm{H}, \mathrm{s}, \mathrm{NH}), 8.95(1 \mathrm{H}, \mathrm{m}, \mathrm{NH}), 8.00(1 \mathrm{H}, \mathrm{d}, J=7.8 \mathrm{~Hz}, \mathrm{ArH}), 7.85(1 \mathrm{H}, \mathrm{d}, J=7.8$ $\mathrm{Hz}, \mathrm{ArH}), 7.57-7.34$ (4H, m, ArH), 7.12-6.91 (2H, m, ArH), 5.30-5.15(1H, m, NCH), 4.42-4.29 $\left(2 \mathrm{H}, \mathrm{m}, \mathrm{CH}_{2} \mathrm{~S}\right), 3.78$ (2H, m, $\left.\mathrm{CH}_{2} \mathrm{O}\right)$. Anal. Calcd. for $\mathrm{C}_{20} \mathrm{H}_{17} \mathrm{~N}_{5} \mathrm{O}_{4} \mathrm{~S}_{2}$ (455.54): C, 52.73; H, 3.70; $\mathrm{N}$, 15.38. Found C, 52.66; H, 3.76; N, 15.03. Mass spectrum, $m / z(\%): 455$ (23.1), 224 (30.8), 208 (26.9), 207 (42.3), 180 (42.3), 179 (26.9), 167 (100), 166 (50.0), 162 (34.0), 160 (42.3), 146 (23.1), 136 (34.6) 135 (50.0), 134 (23.1), 132 (57.7), 119 (46.2), 118 (50.0), 91 (46.2), 90 (34.6).

2-Benzothiazolylthioacetyl L-methionyl 4'-nitro-benzylidene hydrazone (14a). Pale yellow crystals $(0.34 \mathrm{~g}, 83 \%) \mathrm{R}_{\mathrm{f}}=0.47\left(\mathrm{~S}_{1}\right), \mathrm{mp} 172-175{ }^{\circ} \mathrm{C} .{ }^{1} \mathrm{H} \mathrm{NMR}\left(\mathrm{CDCl}_{3}\right): \delta=10.59(1 \mathrm{H}, \mathrm{s}, \mathrm{NH}$, Z-form), 9.91 (1H, s, NH, $E$-form), 8.56 (1H, d, $J=8.2 \mathrm{~Hz}, \mathrm{NH}, Z$-form), $8.42(1 \mathrm{H}, \mathrm{d}, J=8.2 \mathrm{~Hz}$, $\mathrm{NH}, E$-form) 8.23-8.04 (2H, m, ArH), 7.94-7.72 (5H, m, 4ArH, CH), 7.45-7.31 (2H, m, ArH), 5.60-5.54 (1H, m, NCH, E-form), 4.80-4.68 (1H, m, NCH, Z-form), 4.14-3.92 (2H, m, $\left.\mathrm{CH}_{2} \mathrm{~S}\right)$, 2.53-2.42 (2H, m, $\left.\mathrm{CH}_{2} \mathrm{~S}\right), 2.26-2.01\left(2 \mathrm{H}, \mathrm{m}, \mathrm{CH}_{2}\right), 1.94$ (3H, s, $\mathrm{SCH}_{3}, \mathrm{Z}$-form), 1.89 (3H, s, $\mathrm{SCH}_{3}$, E-form). Anal. Calcd. For $\mathrm{C}_{21} \mathrm{H}_{21} \mathrm{~N}_{5} \mathrm{O}_{4} \mathrm{~S}_{3}$ (504.10): C, 50.08; H, 4.21; N, 13.91; Found C, 50.12; H, 4.16; N, 13.61\%. Mass spectrum, $m / z$ (\%): 505 (3.1), 504 (2.6), 322 (15.4), 208 (4.6), 207 (4.1), 181 (5.6), 167 (7.2), 166 (6.2), 150 (29.8), 137 (9.2), 123 (38.5), 122 (10.3), 77 (100), 76 (28.7).

2-Benzothiazolylthioacetyl L-methionyl 4'-methoxy-benzylidene hydrazone (14b). Colorless crystals $(0.33 \mathrm{~g}, 82.4 \%), \mathrm{R}_{\mathrm{f}}=0.68\left(\mathrm{~S}_{1}\right), \mathrm{mp} 184-186{ }^{\circ} \mathrm{C} .{ }^{1} \mathrm{H} \mathrm{NMR}\left(\mathrm{CDCl}_{3}\right): \delta=10.19(1 \mathrm{H}, \mathrm{s}, \mathrm{NH}$, $Z$-form), 9.39 (1H, s, NH, $E$-form), 8.49 (1H, d, $J=8.2 \mathrm{~Hz}, \mathrm{NH}, Z$-form), 8.36 (1H, d, $J=8.2 \mathrm{~Hz}$, $\mathrm{NH}, E$-form), 7.97-7.90 (2H, m, ArH), 7.75 (1H, s, CH, Z-form), 7.71 (1H, s, CH, E-form), 7.607.54 (2H, m, ArH), 7.40-7.29 (2H, m, ArH), 6.91-6.80 (2H, m, ArH), 5.60-5.55 (1H, m, NCH, Eform), 4.78-4.66 (1H, m, NCH, Z-form), 4.16-3.86 (2H, m, $\left.\mathrm{CH}_{2} \mathrm{~S}\right), 3.83\left(3 \mathrm{H}, \mathrm{s}, \mathrm{OCH}_{3}, E\right.$-form), $3.80\left(3 \mathrm{H}, \mathrm{s}, \mathrm{OCH}_{3}, \mathrm{Z}\right.$-form $), 2.47-2.38\left(2 \mathrm{H}, \mathrm{m}, \mathrm{CH}_{2} \mathrm{~S}\right), 2.29-2.05\left(2 \mathrm{H}, \mathrm{m}, \mathrm{CH}_{2}\right), 1.94(3 \mathrm{H}, \mathrm{s}$, 
$\mathrm{CH}_{3} \mathrm{~S}, \mathrm{Z}$-form), 1.85 (3H, s, $\mathrm{CH}_{3} \mathrm{~S}, E$-form). Anal. Calcd. for $\mathrm{C}_{22} \mathrm{H}_{24} \mathrm{~N}_{4} \mathrm{O}_{3} \mathrm{~S}_{3}$ (488.68): C, 54.07; $\mathrm{H}, 4.96 ; \mathrm{N}, 11.47$. Found C, 53.98; H, 4.89; N, 11.33\%. Mass spectrum, $m / z(\%): 488(8.6), 487$ (7.1), 414 (40.0), 413 (28.6), 225 (31.6), 224 (20.0), 209 (25.7), 208 (100), 207 (32.9), 180 (64.3), 179 (25.7), 167 (94.3), 166 (92.9), 136 (44.3), 135 (80.0), 134 (77.1), 108 (45.7).

2-Benzothiazolylthioacetyl L-methionyl 4'-hydroxy-3'-methoxy-benzylidene hydrazone (14c). Colorless crystals $(0.28 \mathrm{~g}, 67.8 \%), \mathrm{R}_{\mathrm{f}}=0.43\left(\mathrm{~S}_{1}\right), \mathrm{mp} 198-200{ }^{\circ} \mathrm{C} .{ }^{1} \mathrm{H} \mathrm{NMR}\left(\mathrm{CDCl}_{3}\right): \delta=$ 10.88 (1H, s, NH, Z-form), $10.66(1 \mathrm{H}, \mathrm{s}, \mathrm{NH}, E$-form), 8.49 (1H, d, J=8.4 Hz, NH, Z-form), $8.17(1 \mathrm{H}, \mathrm{s}, \mathrm{CH}, E$-form), $8.36(1 \mathrm{H}, \mathrm{d}, J=8.4 \mathrm{~Hz}, \mathrm{NH}, E$-form), 7.78-7.55 (2H, m, ArH), 7.59 (1H, s, CH, Z-form), 7.26-7.10 (3H, m, ArH), 6.82-6.62 (2H, m, ArH), 5.39-5.85 (1H, m, NCH, $E$-form), 4.58-4.47 (1H, m, NCH, Z-form), 3.97-3.73 (2H, m, $\left.\mathrm{CH}_{2} \mathrm{~S}\right), 3.70\left(3 \mathrm{H}, \mathrm{s}, \mathrm{OCH}_{3}, E-\right.$ form), $3.68\left(3 \mathrm{H}, \mathrm{s}, \mathrm{OCH}_{3}, \mathrm{Z}\right.$-form $), 2.67(1 \mathrm{H}, \mathrm{s}, \mathrm{OH}), 2.31-2.21\left(2 \mathrm{H}, \mathrm{m}, \mathrm{CH}_{2} \mathrm{~S}\right), 2.00-1.82(2 \mathrm{H}$, $\left.\mathrm{m}, \mathrm{CH}_{2}\right), 1.47\left(3 \mathrm{H}, \mathrm{s}, \mathrm{CH}_{3} \mathrm{~S}, Z\right.$-form $), 1.67\left(3 \mathrm{H}, \mathrm{s}, \mathrm{CH}_{3} \mathrm{~S}, E\right.$-form $)$. Anal. Calcd. for $\mathrm{C}_{22} \mathrm{H}_{24} \mathrm{~N}_{4} \mathrm{O}_{4} \mathrm{~S}_{3}$ (504.68): C, 52.35; H, 4.80; N, 11.10. Found, C, 52.42; H, 4.77; N, 11.03. Mass spectrum, $m / z$ (\%): 504 (3.3), 430 (20.5), 224 (19.2), 208 (66.9), 207 (45.0), 180 (37.1), 179 (25.8), 167 (100), 166 (50.3), 165 (30.5), 150 (29.8), 136 (35.1), 123 (82.8).

2-Benzothiazolylthioacetyl L-serinyl 4'-nitro-benzylidene hydrazone (14d). Pale yellow crystals $(0.31 \mathrm{~g}, 73.2 \%), \mathrm{R}_{\mathrm{f}}=0.25\left(\mathrm{~S}_{1}\right), \mathrm{mp} 214-6^{\circ} \mathrm{C}$. IR $(\mathrm{KBr}): 3536(\mathrm{OH}), 3255(\mathrm{NH}), 1679$, 1650 ( $\mathrm{C}=\mathrm{O}$ amide). Anal. Calcd. for $\mathrm{C}_{19} \mathrm{H}_{17} \mathrm{~N}_{5} \mathrm{O}_{5} \mathrm{~S}_{2}$ (459.53): C, 49.67; H, 3.73; N, 15.24. Found $\mathrm{C}, 49.74 ; \mathrm{H}, 3.71 ; \mathrm{N}, 14.78 \%$. Mass spectrum, $\mathrm{m} / z$ (\%): 459 (15.0), 458 (15.0), 250 (22.5), 208 (55.0), 207 (27.5), 181 (65.0), 180 (45.0), 179 (52.5), 168 (35.0), 167 (100), 166 (90.0), 164 (45.0), 149 (37.5), 148 (32.5) 136 (35.0), 135 (32.5), 123 (37.5), 122 (35.0), 108 (37.5).

2-Benzothiazolylthioacetyl L-serinyl 4'-methoxy-benzylidene hydrazone (14e). Colorless crystals $(0.33 \mathrm{~g}, 79.8 \%), \mathrm{R}_{\mathrm{f}}=0.30\left(\mathrm{~S}_{1}\right), \mathrm{mp} 196-8{ }^{\circ} \mathrm{C}$. IR (KBr): $3385(\mathrm{OH}), 3311(\mathrm{NH}), 3253$ $(\mathrm{NH}), 1664,1602$ (C=O amide). Anal. Calcd. for $\mathrm{C}_{20} \mathrm{H}_{20} \mathrm{~N}_{4} \mathrm{O}_{4} \mathrm{~S}_{2}$ (444.56): C, 54.04; H, 4.54; N, 12.60; Found C, 53.80; H, 4.49; N, 12.32. Mass spectrum, $m / z$ (\%): 444 (18.6), 295 (19.3), 268 (16.9), 225 (14.2), 209 (13.8), 208 (100), 181 (42.2), 180 (65.1), 168 (32.1), 167 (59.4), 166 (19.4), 150 (96.0), 148 (33.4) 136 (49.1), 135 (55.3), 134 (61.3), 120 (31.7), 108 (35.2).

2-Benzothiazolylthioacetyl L-serinyl 4'hydroxy-3'-methoxy-benzylidene hydrazone (14f). Colorless crystals $(0.32 \mathrm{~g}, 74.4 \%), \mathrm{R}_{\mathrm{f}}=0.18\left(\mathrm{~S}_{1}\right), \mathrm{mp} 199-201{ }^{\circ} \mathrm{C}$. IR $(\mathrm{KBr}): 3514(\mathrm{OH}), 3314$ $(\mathrm{NH}), 3266(\mathrm{NH}), 1678,1617$ (C=O amide). Anal. Calcd. For $\mathrm{C}_{20} \mathrm{H}_{20} \mathrm{~N}_{4} \mathrm{O}_{5} \mathrm{~S}_{2}$ (459.53): C, 52.16; H, 4.38; N, 12.16; Found: C, 52.14; H, 4.39; N, 11.96. Mass spectrum, $m / z$ (\%): 460 (14.6), 225(28.3), 208(34.8), 193 (24.7), 181 (100), 180 (69.2), 167 (58.3), 166 (47.3), 151 (27.9), 150 (80.9), 149 (53.4), 148 (58.0), 136 (73.4), 135 (75.7), 123 (32.9), 108 (61.0).

Di-[2-Benzothiazolylthioacetyl L-methionyl]benzylidene-1,4-dihydrazone (15). Colorless crystals $(0.63 \mathrm{~g}, 92.7 \%), \mathrm{R}_{\mathrm{f}}=0.18\left(\mathrm{~S}_{1}\right), \mathrm{mp} 234-236{ }^{\circ} \mathrm{C} .{ }^{1} \mathrm{H}$ NMR (DMSO): $\delta=11.53(2 \mathrm{H}, \mathrm{s}, 2$ $\mathrm{NH}), 8.73(2 \mathrm{H}, \mathrm{d}, J=7.2 \mathrm{~Hz}, 2 \mathrm{NH}), 8.21(2 \mathrm{H}, \mathrm{s}, 2 \mathrm{CH}), 8.01(2 \mathrm{H}, \mathrm{d}, J=7.0 \mathrm{~Hz}, 2 \mathrm{ArH}), 7.85$ $(2 \mathrm{H}, \mathrm{d}, J=7.8 \mathrm{~Hz}, 2 \mathrm{ArH}), 7.74(4 \mathrm{H}, \mathrm{s}, 4 \mathrm{ArH}), 7.46-7.32(4 \mathrm{H}, \mathrm{m}, 4 \mathrm{ArH}), 5.39-5.23(2 \mathrm{H}, \mathrm{m}$, $2 \mathrm{NCH}), 4.48-4.13\left(4 \mathrm{H}, \mathrm{m}, 2 \mathrm{CH}_{2} \mathrm{~S}\right), 2.63-2.40\left(4 \mathrm{H}, \mathrm{m}, 2 \mathrm{CH}_{2} \mathrm{~S}\right), 2.01-1.82\left(10 \mathrm{H}, m, 2 \mathrm{CH}_{2}, 2\right.$ $\mathrm{CH}_{3} \mathrm{~S}$ ). Anal. Calcd. For $\mathrm{C}_{36} \mathrm{H}_{38} \mathrm{~N}_{8} \mathrm{O}_{4} \mathrm{~S}_{6}$ (838.134): C, 51.52; H, 4.56; N, 13.35. Found C, 51.65; $\mathrm{H}, 4.57$; N, 13.23. Mass spectrum, $m / z$ (\%): 841(1.2), 838 (2.3), 615 (21.4), 501 (13.9), 264 
(47.3), 225 (37.5), 209 (17.8), 208 (74.1), 181 (49.8), 180 (100), 168 (31.2), 167 (44.0), 166 (22.8), 136 (16.0), 108 (47.9), 90 (12.2).

\section{Biological assay}

Fungal strains. Clinical isolates of mold fungi (Aspergillus flavus) and yeast (Candida albicans) were used to evaluate the antifungal activity of the synthesized compounds.

Tested compounds. The tested compounds were dissolved in dimethyl sulphoxide to make a parent concentration of $10^{3} \mu \mathrm{g} / \mathrm{ml}$, and the other concentrations were prepared by dilution.

Determination of MIC. A standardized fungal spore suspension $10^{4} \mathrm{CFU} / \mathrm{ml}$ was prepared according to Grande and Artis. ${ }^{28}$ The agar diffusion method (well technique) was employed to determine the approximate minimal inhibitory concentration (MIC) values, the concentration considered inhibitory if minimal inhibition was found around the well.

Determination of MFC. Sterilized test tubes containing standardized fungal spore suspension were incorporated separately with the tested compounds at different concentrations starting from those of the MIC values and gradually multiplied with a control tube incorporated with the highest value of the solvent used as a control and another incorporated with antifungal drug (fluconazole) for comparison. Fungal CFU were counted and their viability was checked at various time intervals from 0 to 24 hours. MFC was recorded as the minimal concentration at which about $99 \%$ of the viable CFU was destroyed.

\section{References}

1. De Wever, H.; Verachtert, H. Wat. Res. 1997, 31, 2673.

2. Zhang, Y.; Zhong Qiao, R.; Feng Dai, C.; Fei Xu, P.; Zhang, Z. Y. Chin. Chem. Lett. 2002, 13, 287.

3. Siddiqui, N.; Rana, A.; Khan, S. A. Indian J. Pharm. 2007, 69, 10.

4. Metzger, J. V. In Comprehensive Heterocyclic Chem. Chapter on Thiazole and Benzothiazole Derivatives Katritzky, A.; Rees, C. W., Eds. Pergamon, 1984, Vol.6 pp 330331

5. Bujdakova, H.; Muckova, M. Int. J. Antimicrob. Agents 1994, 4, 303.

6. Blockinger, G.; Furdik, N.; Schwarz, E.; Moys, A. Acta F. R. N. Univ. Comen., Chimia. 1968, 12, 293.

7. Bujdakova, H.; Kuchta, T.; Sidoova, E.; Gvozdjakova, A. FEMS Microbiol. Lett. 1995, 112, 329.

8. Bujdakova, H.; Kralova, K.; Sidoova, E. Pharmazie. 1994, 49, 375. 
9. Bujdakova, H.; Muckova, M.; Klobusicky, M.; Sidoova, E. Mycopathologia. 1995, 130, 141.

10. Bujdakova, H.; Kralova, K.; Sidoova, E. Pharmazie 1995, 50, 156.

11. Feignier, C.; Besson, F.; Hoet, P.; Di Giambattista, M.; Cocito, C.; Sabatier, C.; Michel, G. Biotechnol. Tech. 1993, 7, 423.

12. Eshita, S. M.; Roberto, N. H. J. Antibiot. 1995, 48, 1240.

13. Walsh, T. J.; Giri, N. Eur. J. Clin. Microbiol. Infect. Dis.1997, 16, 93.

14. Delucca, A. J.; Walsh, T. J. Rev. Iberoam Micol. 2000, 17, 116.

15. Carrillo-Munoz, A. J.; Giusiano, G.; Ezkurra, P. A.; Quindos, G. Rev. Esp. Quimioterap. 2006, 19, 130.

16. Yoon, Y.; Lee, C. Biotechnol. Bioprocess Eng. 2009, 14, 383.

17. Sen, M.; Meshra, N.; Nayak, A. J. Indian Chem. Soc. 1990, 67, 409.

18. Hofmann, K.; Johl, A.; Furlemeier, A. E.; Koppeler, H. J. J. Am. Chem. Soc. 1957, 79, 1636.

19. Hofmann, K.; Thompson, T. A.; Yajima, H.; Schwarts, E. T.; Enouye, H. J. Am. Chem. Soc. 1960, 82, 3715.

20. Bodansky, M. Principles of Peptide Synthesis, $2^{\text {nd }}$ Edn.; Springer: Berlin, 1993; p 20.

21. Schmidt, R. R.; Michel, J. Angew. Chem. 1980, 92, 763; Angew. Chem. Int. Ed. Engl. 1980, 19, 731 .

22. Kaymakcioğlu, B. K.; Oruc-Emre, E. E.; Unsalan, S.; Rollas, S. Med. Chem. Res. 2009, 18, 277.

23. Rutavichyus, A.; Valiulene, S.; Kuodis, Z. Chem. Heterocycl. Compd. 2000, 36, 851.

24. Brokaite, K.; Mickevicius, V.; Mikulskiene, G. Arkivoc 2006, ii, 61.

25. Abdel Gany Hamed Ahmed, M. Sc. Thesis, "Synthesis and reactions of some benzothiazole derivatives with Schistosomicidal / Molluscicidal activities"; Faculty of Science, Suez Canal University 2000.

26. Schmidt, O. T. Methods Carbohydr. Chem. 1963, 2, 318.

27. Bekircan, O.; Bektas, H. Molecules. 2008, 13, 2126.

28. Grande, T. C.; Artis, W. M. Antimicrobial Agents Chemotherapy. 1980, 17, 725 